\title{
THE UNIVERSITY OF BRITISH COLUMBIA
}

FACULTY OF GRADUATE STUDIES

\section{PROGRAMME OF THE.}

\section{FINAL ORAL EXAMINATION FOR THE DEGREE OF DOGTOR OF PHILOSOPHY}

\author{
of \\ PHILIP NORMAN DAYKIN \\ B.A. (University of British Columbia) 1947 \\ M.A. (University of British Columbia) 1949
}

\begin{abstract}
WEDNESDAY, APRIL 23rd, 1952, at 3:00 P.M. IN ROOM 303, PHYSICS BUILDING
\end{abstract}

\section{Commetre In Chakge:}

Dean H. F. Angus, Chairman

\author{
l'rofessor F. A. Kaempfier \\ Professor W. A. Clemens \\ Professor R. W. Wellwood \\ Jrofessor CG. M. Shrum \\ Prof cssor R. Daniells \\ l'rofessor R. D. James \\ Prolessor D. G. Laird
}




\section{PUBLISHED PAPERS}

Radiations from Zinctsis, Physical Revicw 76, 1719, 1949 (Co-authors K. C. Mann and D. Rankin)

An Analysis of the Self-Energy Problem for the Frec Resting Electron, Physical Review 83, 895 (A) , 1951 (Co-author F. A. Kacmplfer) (Read to the American Physical Society)

Conservation Laws in Feynman's Modified. Electrodynamics, Physical Review 83, 986 (A) , 1951. (Read to the American I'hysical Society)

Conservation Laws in Feynman's Modified Electrodynamics, Canadian Journal of Physics, 29, 459, 1952

An Analysis of the Self-Energy Problem for the Electron in Quaniun Electrodynamics, Canadian Journal of Physics, 30, 70, 1952 


\section{THESIS}

\section{AN ANALYSIS OF THE SELF-ENERGY PROBLEM FOR THE ELEC'TRON IN QUANTUM ELECTRODYNAMICS"}

The self-energy of the free electron at rest is evaluated without the restriction that the self-interaction be a purely retarded interaction. Both the one-electron theory and the hole theory of the positron are treated. It: is shown that in the one-electron theory the normally quadratically divergent transierse part of the self-energy vanishes if the self-interaction is assumed to be one half retarded plus one half advanced, the remaining Cotilomb part of the self-energy being only linearly divergent. A similar theorem does not hold for the hole theory. A particular type of self-interaction leads to a vanishing self-energy in:oneelectron theory. However this does not solve the self-energy problem, as in this case radiation corrections to scattering will vanish as well.

The self-energy of a bound electron is evaluated in a similar manner. The decay probability of an excited state is calculated as the imaginary part of the self-energy; the correct value is obtained only for a purely retarded self-interaction in hole theory. In the special case in which the external field is a uniform magnetic field, again only, this interaction in hole theory gives the correct value for the anomalous magnetic moment.

It is therefore concluded that any solution of the self-energy problem by introducing advanced self-interactions is to be ruled out. 


\section{GRADUATE STUDIES}

Field of Study: Physics

Spectroscopy-l'rof. A. M. Crooker

Relativity-Prof. F. J. Belinfante

Wave Mechanics-Prof-F. J. Belinfante-

Beta Ray Spectroscopy-Prof:-K. C. Mann

Electromagnetic Theory-Prof. G. L. Pickard

Nuclear Physics-P'rof.. K. C. Mann

Electronics-Prof. A, van der Ziel

Chemical Physics-Prof. A. J. Dekker

Quanum Theory of Molccules and Solids-l'rof. H. M. James

Statistical Mechanics-Prof: H.,Frohlich

Quantum Theory of Radiation-Pref. F. A. Kaempfler

Theoretical Physics Seminar-P'rof. W. Opechowski

\section{Other Studies:}

Advanced Differential Equations-Dean W. H. Gage "'

Tensón Calculus-Dean. W. H. Gage

Theory of Functions of a Real Variable-l'rof. S. A. Jennings

Group Theory-Prof. S. A. Jennings

Integral Equations-Prof. T. E. Hull

Modern Algebra-Prof. D. Derry 
AN. ANAIYSIS OF THE SELF-ENERGY PROBLEM

FOR THE EIECTRON IN QUANTUM ELECTRODYNAMICS

by

PHIIIP NORMAN DAYKIN

A THESIS SUBMITTED IN PARTIAL FULFILMENT OF THE REQUIREMENTS FOR THE DEGREE OF

DOCTOR OF PHILOSOPHY

IN

PHYSICS

We accept this thesis as conforming to

the standard required from candidates

for the degree of DOCTOR OF PHILOSOPHY

Members of the Department of Physics

THE UNIVERSITY OF BRITISH COLTMBIA

APRIL, 1952 
The self-energy of the free electron at rest is evaluated without the restriction that the self-interaction be a purely retarded interaction. Both the one-electron theory and the hole theory of the positron are treated. It is shown that in the one-electron theory the normally quadratically divergent transverse part of the self-energy vanishes if the self-interaction is assumed to be one half retarded plus one half advanced, the remaining Coulomb part of the self-energy being only linearly divergent. A similar theorem does not hold for the hole theory. A particular type of self-interaction leads to a vanishing self-energy in one-electron theory. However this does not solve the self-energy problem, as in this case radiation corrections to scattering will vanish as well.

The self-energy of a bound electron is evaluated in a similar manner. The decay probability of an excited state is calculated as the imaginary part of the self-energy; the correct value is obtained only for a purely retarded selfinteraction in hole theory. In the special case in which the external field is a uniform magnetic field, again only this interaction in hole theory gives the correct value for the anomalous magnetic moment.

It is therefore concluded that any solution of the selfenergy problem by introducing advanced self-interactions is to be ruled out. 


\section{ACKNOWLEDGEMENTS}

This research was carried out with the aid of a Fellowship from the National Research Council.

The author wishes to thank Professor F.A. Kaempfer for suggesting the problem and for numerous helpful discussions during the investigation. 


\section{SECTION I}

Introduction

SECTION II

Retarded and Advanced Interactions Between. Two Dirac Particles

SECTION III

Evaluation of the Free Electron Self-Energy Matrix

SECTION IV

Self-Energy Matrix For An Electron In An External Field

SECTION $V$

The Anomalous Magnetic Moment of the Electron

SECTION VI

The Retarded Field of a Free Rest Electron

A. Number of Photons in the Virtual Field

B. The Electric and Magnetic Fields of a Rest Electron38 SECTION VII

Appendix

- A. The Anomalous Magnetic Moment

B. The Photon Number

C. The Transverse Fields

D. The Coulomb Field

SECTION VIII 


\section{INTRODUCTION}

The self-energy problem for the electron in quantum electrodynamics arises from the coupling between one electron and the total radiation field. According to the formalism of perturbation theory the electron can emit and reabsorb photons. Thus, the system consisting of one electron and the total radiation field can exist partly in intermediate states which contain virtual photons and in which the electron has an additional recoil momentum. The self-energy of the electron is the average value over the intermediate states of three terms: the kinetic energy of the electron, the energy of the virtual photons and the energy of the coulomb field of the electron. The first two terms combined give the transverse self-energy; the third the longitudinal or coulomb self-energy. Both parts, of the self-energy are divergent: the transverse part from the large contribution from intermediate states with high kinetic energy; the coulomb part, which is analogous to the classical self-energy, essentially because the electron must be treated as a point charge.

The self-energy for a free electron was first calculated relativistically by Waller $(1,1930)$ using the one-electron theory of Dirac $(2,1928)$ and the quantum theory of radiation developed by Dirac (B, 1927) and by Heisenberg and Pauli (4, 1929). The coulomb self-energy was shown to be linearly divergent as for a classical point charge but the transverse self-energy was 
quadratically divergent.

The reason for the quadratic divergence is connected with the difficulty of the negative energy states in the one-electron theory. For transitions to the low lying states energy is very nearly conserved and so these states contribute largely to the self-energy. In fact, as shown in Section VI the number of virtual photons is quadratically divergent in the one-electron theory.

Oppenheimer $(5,1930)$ extended the radiation theory to the problem of a bound electron. The transverse self-energy in second order was shown to have the form

$$
E_{m}^{(2)}=-\int d v_{r} \int d \omega_{r} \sum_{\lambda} \sum_{n} \frac{\left|b_{m n}^{r \lambda}\right|^{2}}{h \nu_{r}+E_{n}^{(0)}-E_{m}^{(0)}}
$$

where $b_{m n}^{r_{\lambda}}$ is the matrix element for emission of a photon of frequency $\nu_{r}$ and polarization $\lambda$ with corresponding transition of the electron from the state $m$ to state $n$. The integration extends over all solid angle $d w_{r}$ and frequence $v_{r}$. The improper integral over $\forall$ is resolved by deforming the path of integration about the pole. This adds an imaginary term

$$
\pm \pi i \delta\left(h \nu_{r}+E_{n}^{(0)}-E_{m}^{(0)}\right)
$$

to the self-energy for $E_{n}^{(0)}<E_{m}^{(0)}$ which correctly accounts for the half width of the spectral lines. However the center of the spectral line, it was shown, would be shifted toward the red by the difference between the self-energies $E_{m}^{(2)}$ and $E_{n}^{(2)}$ and this difference would in general also be infinite.

The hole theory of the positron formulated by Dirac $(6,1929)$ 
gave rise to some hopes that the degree of divergence could be reduced. Weisskopf $(7,1934 ; 8,1939)$ showed in fact that the self-energy relative to the vacuum was only logarithmically divergent. Briefly, the reasons for the divergence being only logarithmic in hole theory are two-fold. Firstly, the quadratic divergence of the one-electron: theory is reduced to a linear divergence by exclusion of transitions to the negative energy states. Secondly, in the neighborhood of the electron virtual pairs are created which in effect produce a spread of charge over a region of radius equal to the Compton wavelength. This spread of charge is sufficient to make the self-energy logarithmically divergent. However, the conclusion of oppenheimer regarding the predicted infinite shift in the frequencies of atomic spectral lines remained valid. The self-energy in quantum electrodynamics was therefore discarded, as in the classical theory, as meaningless.

During the three years 1937 to 1940 some spectroscopic evidence was accumulating which indicated that the Dirac equation did not predict exactly the correct fine structure of atomic states (9): Pasternack (10) showed that the observed fine structure of the $H_{\alpha}$ line could be accounted for by a shift of only the $2 S_{\frac{1}{2}}$ level by $.03 \mathrm{~cm}^{-1}$ from the value predicted by the Dirac equation. A number of authors discussed the effects of a postulated departure from the coulom: $b$ field. In all cases the necessary departure was much too large to be reasonable. These doubts concerning Dirac's fine structure formula were confirmed by the brilliant experiments of Lamb and Retherford (11, 1947, 1949) whIch showed conclusively 
that the $2 \mathrm{P} \frac{1}{2}$ and $2 \mathrm{~S} \frac{1}{2}$ spectroscopic states of the hydrogen atom were not degenerate as predicted by the Dirac equation but were separated by some 1000 megacycles per second.

It was shown by a number of authors that this discrepancy could be accounted for in the following way by the self-energy terms which had earlier been discarded. The self-energy of a free electron is proportional to the mass and may be regarded as a contribution to the electron's kinetic energy from addition of electromagnetic mass to the mechanical mass. However this electromagnetic mass, $\Delta \mathrm{m}$, must already be included in the total observed mass, $m$, and should formally be put equal to zero. This argument is equivalent to assuming that the original mass appearing in the Dirac equation was the mechanical mass and that this should now be replaced by the total mass. The same renormalization of mass must now be performed on the self-energy of a bound electron. A term equal to the average value of $(\mathrm{dH} / \mathrm{dm}) \Delta \mathrm{m}$ for the atomic state should be subtracted. The remaining finite part should give the observed term shift. It should be emphasized that this procedure can only be justified on the basis that future modifications of the theory for high energy photons will make the electromagnetic mass a small correction.

A preliminary non-relativistic calculation by Bethe $(12,1947)$ gave nearly the observed Lamb shift. Relativistic corrections for the intermediate states with high kinetic energy by a number of authors (13, 1949, 1950) gave results in good agreement with the 
more accurate measurements of Lamb and Retherford (1949). It was pointed out by Schwinger (14, 1948) that one of the terms in the relativistic expression for the Lamb Shift could be explained on the basis of a small correction to the magnetic moment of the electron of magnitude $\alpha / 2 \pi$ Bohr magnetons. The existence of this anomalous magnetic moment had earlier been suggested by Breit $(15,1947)$ on the basis of spectroscopic data.

A. number of physically equivalent Lorentz-covarlant formalisms were developed to deal with the problem of radiation corrections to scattering by Tomonaga, Schwinger, Dyson and Feynman (16-21, 1946-50). These had the advantage over the older perturbation theory that the terms equivalent to a renormalization of mass can be separated out from observable effects unambiguously." Feynman's formalism has an added advantage of simplicity in application; Feynman has derived a set of rules which enable the matrix elements to be written down at once unambiguously from the Feynman scattering diagrams. Feynman's method differs from others in another respect: a relativistic cut-off is introduced to make the integrals finite. It has been shown (12) that this is equivalent to modifying the Maxwell equations in the manner described by Podolsky, Bopp and others. After renormalization the observable radiation effects are only slightly sensitive to the cutoff.

A confirmation of the correctness of the new formalismsefor fourth order has recently appeared in the measurement of the anomalous magnetic moment of the electron. Koenig, Prodell and 
Kusch $(18,1951)$ found experimentally for the g-factor of the electron the value $2(1.0011450 .000013)$ which agrees with the theoretical valiue of $2(1.0011454)$ calculated by Karplus and Kroll (19, 1950) using Dyson's S-matrix.

These well known calculations are based on the assumption, either explicit or implicit, that the interaction between charges (which includes the self-interaction of a charge as a special case) is a retarded interaction. In quantum electrodynamics a retarded interaction is described by the exchange of positive energy photons, as Feynman (2I) has shown by elimination of the field oscillators. However, there is up to now no conclusive experimental evidence that this assumption is correct. In fact wheeler and Feynman (25) have shown in their absorber theory of radiation that a consistent classical description of electromagnetic phenomena including radiation damping can be obtained if the radiation field of a charge is assumed to be one half of the retarded plus one half of the advanced fleld.

Dirac (26) has proposed a relativistic quantum theory of radiation which treats positive and negative energy photons symmetrically. It will be shown in Section II that this treatment is equivalent to the assumption that the interaction between electrons is one half retarded plus one half advanced, corresponding to the classical Wheeler-Feynman interaction between charges. Pauli (27) has shown that this treatment leads to a vanishing transverse part of the free electron self-energy in one-electron theory, but if the hole theory of the positron is adopted a similar 
theorem does not hold. Addition of the effects of positive and negative energy photons in fact destroys the logarithmic character of the divergence, leaving quadratically divergent terms. Muto and Inone (28) report, however, that the divergence in the fourth order self energy in one-electron theory is not, eliminated by introducing negative energy photons. In referring to these results the effects of the $\lambda$-limiting process, which is also included in Dirac's method of field quantization, have been neglected. This process results in introducing a factor cos $\lambda$ k into the integrals over photon energy, $\mathrm{k}$, and leads to a vanishing coulomb part of the self energy in one-electron theory but does not improve the result in hole theory. Moto and Inone have shown that the energy level shift of the $25 \frac{1}{2}$ state of the hydrogen atom vanishes if both the $\lambda$-limiting process and the negative energy photons are included.

It is the main purpose of this thesis to investigate the self-energy problem without the restriction that the interaction of a charge with itself be purely retarded. For this investigation use has been made of the simplified form of quantum electrodynamics described by Feynman $(19,20)$. It is shown that the distinction between one-electron theory and hole theory and between the different types of interaction can be made by defining different contours for evaluating the Fourier transforms of the singular solutions of the Maxwell equations and Dirac equations, in terms of which the interaction between electrons is defined. The meaning of the different types of interaction is illustrated 
by a discussion of the second-order interaction between two electrons. A Hamiltonian can be obtained for each type of interaction as an expansion in powers of $\mathrm{v} / \mathrm{c}$ up to the second. This is compared with the Breit interaction (29). Higher order terms in $\mathrm{v} / \mathrm{c}$ are in general not hermitian, the antihermitian parts corresponding to the finite life time of two-electron states without radiation. However, if the interaction is assumed to be one-half retarded plus one-half advanced, the matrix of the interaction is hermitian to all powers in $\mathrm{v} / \mathrm{c}$ in accordance with the wheelerFeynman theory.

In the self-energy problem for a free electron Pauli's results are verified for the half retarded plus half advanced self interaction. In addition it is found that there exists a particular type of interaction corresponding to a certain singular solution of the Maxwell equations discussed by Stueckelberg (30) which leads to a vanishing self-energy in one electron theory. However, this does not represent a satisfactory solution of the self-energy problem as in this case the radiation corrections to scattering would vanish as well. Thus it would be impossible to obtain theoretically expressions for experimentally observed effects like the Lamb shift and anomalous magnetic moment of the electron.

In the case of a bound electron the modified Dirac equation for an electron interacting with itself is obtained which is similar in form to that given by schwinger (31). The self-energy is then evaluated for the same contours as used in the free 
electron case. The self-energy in general is not real; the negative of the imaginary part gives the decay probability per unit time for the initial bound state. It is found that the correct decay probability arises from only one contour, the contour which corresponds to hole theory and a retarded interaction.

The self-energy is evaluated for the case of an electron in a weak homogeneous magnetic field following the method of Luttinger (32). Again it is only the retarded interaction in hole theory that gives the correct value of the anomalous magnetic moment.

In section VI the virtual field of a free rest electron is investigated for the purpose of determining the character of the divergence of the field strengths and of the total energy of the field. Only the retarded field of the electron is calculated. It is shown that in one-electron theory the electric field is just the Coulomb field of a point charge and the magnetic field is the field of a "point" magnetic dipole. This result reveals the origin of the divergence of the self-energy. In hole theory the vacuum polarization causes a spread in the charge and dipole densities over a region whose dimension is of the order of the Compton wavelength. This spread is sufficient to reduce the order of divergence of the field strengths at the center of the charge distribution but not to eliminate the divergence completely. The average number of photons in the field is evaluated in one-electron theory and in hole theory. In oneelectron theory the number is quadratically divergent while in 
hole theory it vanishes. These results are in agreement with those of Weisskopf (8) who calculated the transverse electric and magnetic field energies separately. It was shown that in one electron theory these energies were equal and cubically divergent while in hole theory they were equal in magnitude but with opposite sign. 
II. RETARDED AND ADVANCED INTERACTIONS BETWEEN TWO DIRAC PARTICLES

The notation of Feynman (20) will be used throughout this section. Feynman has shown that the kernel or Green's function for two interacting electrons $\mathrm{a}$ and $\mathrm{b}$ may be approximated to first order in $e^{x}$ by

$$
\begin{aligned}
K(3,4 ; 1,2)= & K_{+a}(3,1) K_{+b}(4,2) \\
& -i e^{2} \iint K_{+a}(3,5) K_{+b}(4,6) \gamma_{a \mu} \gamma_{b \mu} \delta_{t}\left(s_{s b}^{2}\right) K_{+a}(5,1) K_{+b}(6,2) d^{4} x_{5} d^{4} x_{b}
\end{aligned}
$$

where $K_{+}(2, I)$ is the kernel for the propagation of a free electron from space time point 1 to point 2 which satisfies the inhomogeneous Dirac equation

$$
\left(p_{2}-m\right) K_{+}(2,1)=i \delta(2, i) \quad ; \quad \underline{p}_{2}=i \gamma_{\mu} \frac{\partial}{\partial x_{\lambda \mu}}
$$

and $\delta_{+}\left(s_{21}^{2}\right)$ is the kernel for the propagation of a (positive energy)photon between $I$ and 2 which satisfies the unhomogeneous Maxwell equation

$$
\square_{2}^{2} \delta_{1}\left(s_{21}^{2}\right)=-4 \pi \delta(2,1) \quad ; \quad s_{21}^{2}=\left(t_{2}-t_{1}\right)^{2}-\left(r_{2}-r_{1}\right)^{2}
$$

These kernels can be defined by the Fourier integrals

$$
\begin{array}{ll}
K_{+}(2,1)=\frac{i}{4 \pi^{2}} \int \frac{d^{4} p}{p-m+c \epsilon^{2}} e^{-i p \cdot x_{21}} \quad ;\left(\underline{p-1 n)^{-1}}=(p+m)\left(p^{2}-m^{2}\right)^{-1}\right. \\
\delta_{+}\left(s_{21}^{2}\right)=-\frac{1}{\pi} \int \frac{d^{4} k}{k^{2}+i \epsilon^{2}} e^{-i k \cdot \lambda_{2},} \quad ; k^{2}=k_{4}^{2}-\vec{k}^{2}
\end{array}
$$

where $\epsilon$ is a real infinitesimal which is introduced to locate the poles of the integrand. It is assumed that the integration will always be carried out first over $p_{4}$ or $k_{4}$.

Thus, equation (1) describes the propagation of two 
electrons with an exchange of one (positive energy) photon. As will be shown below the second term in (I) corresponds to $d$. retarded interaction between a and $b$. However, this is not the only interaction which is consistent with the Maxwell equations. one could substitute for the $\delta_{+}$function any other solution of (3); that is, any function whose fourier transform is $\mathrm{k}^{-2}$. The distinction between such functions lies in the convention for locating the poles of $\mathrm{k}_{4}^{-2}$ relative to the real $k_{4}$-axis. There exist four possibilities for locating the poles $k_{4}^{\prime}=-K$ and $k_{4}^{\prime \prime}=+K$ which are listed in Table I together with the corresponding photon kernels and the type of interaction between charges.

The term "photon kernel" was introduced in analogy with Feynman's "électron kernel". 


\section{TABLE I}

Position of poles $\mathrm{k}$ and $\mathrm{k}$ relative to the real $k$ axis

$k$ above

$$
\begin{aligned}
& \delta_{+}\left(s_{56}^{2}\right)=\frac{1}{2 r_{56}}\left\{\delta_{+}\left(t_{56}-r_{56}\right)+\delta_{+}\left(-t_{56}-r_{56}\right)\right\} \\
& =\frac{1}{2 r_{56}} \int_{0}^{\infty} \frac{d K}{\pi}\left\{e^{-i K\left(t_{56}-r_{56}\right)}+e^{-i k\left(-t_{56}-r_{56}\right)}\right\}
\end{aligned}
$$

Corresponding photon kernel

$k$ below
Type of interaction between charges

Retarded action of $a$ on $b$ plus retarded action of $b$ on $a$

$k$ below

$$
\begin{aligned}
& \delta_{-}\left(s_{56}^{2}\right)=\frac{1}{2 r_{56}}\left\{\delta-\left(t_{56}-r_{56}\right)+\delta_{-}\left(-t_{56}-r_{56}\right)\right\} \\
& =\frac{1}{2 r_{56}} \int_{0}^{\infty} \frac{d K}{\pi}\left\{e^{i K\left(t_{56}-r_{56}\right)}+e^{i K\left(-t_{56}-r_{56}\right)}\right\}
\end{aligned}
$$

Advanced action of $a$ on $b$ plus

$k$ above advanced action of $b$ on $a$

$k$ below

$k$ below

$$
D_{7}\left(x_{56}\right)= \begin{cases}\frac{1}{r_{56}} \delta\left(t_{56}-r_{56}\right) & ; \quad t_{5}>t_{6} \\ 0 & ; t_{5}<t_{6}\end{cases}
$$

Retarded action of $a$ on $b$ plus advanced action of $b$ on $a$

$\mathrm{k}$ above

$$
D_{-}\left(x_{56}\right)=\left\{\begin{array}{lll}
0 & ; t_{5}>t_{6} & \text { Advanced action } \\
\frac{1}{r_{56}} \delta\left(t_{56}-r_{56}\right) ; t_{5}<t_{6} & \text { retarded action } \\
& \text { of } b \text { on a }
\end{array}\right.
$$


The following relations hold between the kernels

$$
\delta\left(s_{s 6}^{2}\right)=\frac{1}{2}\left(\delta_{+}\left(s_{s 6}^{2}\right)+\delta_{-}\left(s_{s 6}^{2}\right)\right)=\frac{1}{2}\left(D_{+}\left(x_{s 6}\right)+D_{-}\left(x_{s 6}\right)\right)
$$

It can be seen from Table I that the Fourier integral representations of the $\delta_{+}\left(s_{s 6}^{2}\right)$ and $\delta_{-}\left(s_{s}^{2}\right)$ photon kernels differ only in the sign of the photon energy. $K$. Thus, Dirac's suggestion that the positive and negative energy photons be treated symmetrically corresponds to the assumption of a Wheeler-Feynman $\delta\left(s_{s_{4}}^{2}\right)$-interaction between charges which, as shown below, is a half retarded plus half advanced interaction. This point will be illustrated again in the self-energy problem.

The entries in Column 3 will now be explained. Following a suggestion of Feynman the second term of (1) may be written as the scattering of $b$ :

$$
K_{b}^{\prime}(4,2)=-i \int K_{+b}(4,6) \gamma_{b \mu} A_{\mu}^{a}(6) K_{+b}(6,2) d^{4} x_{b}
$$

by the potential

$$
\left.A_{\mu}^{a}(6)=\frac{e^{2}}{2} \int \frac{d_{+}\left(-t_{56}-r_{56}\right)}{r_{56}} K_{+a}(3,5) \gamma_{a \mu} K_{+a}(5,1) d^{4} x_{5}\right)
$$

plus the scattering of a:

$$
K_{a}^{\prime}(3,1)=-i \int K_{+a}(3,5) \gamma_{a \mu} A_{\mu}^{b}(5) K_{+a}(5,1) d^{4} x_{5}
$$

by the potentia.l

$$
A_{\mu}^{b}(5)=\frac{e^{2}}{2} \int \frac{\delta_{+}\left(t_{56}-r_{56}\right)}{r_{56}} K_{+b}(4,6) \gamma_{b \mu} K_{+b}(6,2) d^{4} x_{b}
$$

It will be shown that $A_{\mu}^{a}(6)$ gives the retarded field at 6 arising from the positive frequency part of the "current" 


$$
J_{\mu}^{a}(5)=e^{2} K_{+a}(3,5) \gamma_{a \mu} K_{+a}(5,1)
$$

produced by a in going from 1 to 3. An analogous statement holds for $A_{m}^{b}(5)$. From (8) and Table I we have

$$
\begin{aligned}
A_{\mu}^{a}(6) & =\frac{1}{2 \pi} \iint_{0}^{\infty} d k e^{-i k\left(t_{65}-r_{65}\right)} \frac{j_{\mu}^{a}(5)}{r_{65}} d^{4} x_{5} \\
& =\frac{1}{2 \pi} \iint_{0}^{\infty} d k e^{-i k\left(t_{6}-r_{65}\right)} \frac{J_{\mu, k}^{a}\left(r_{5}\right)}{r_{65}} d^{3} x_{5}
\end{aligned}
$$

where

$$
J_{\mu, K}^{a}\left(r_{5}\right)=\int_{-\infty}^{\infty} J_{\mu}^{a}(5) e^{i \kappa t_{5}} d t_{5}
$$

If $J_{\mu+}^{a}(5)$ is defined by

$$
J_{\mu+}^{a}(5)=\frac{1}{2 \pi} \int_{0}^{\infty} d K e^{-i k t 5} J_{\mu, k}^{a}\left(r_{5}\right)
$$

Then

$$
A_{\mu}^{a}(6)=\int J_{\mu}^{a}+\frac{\left(r_{5}, t_{6}-r_{56}\right)}{r_{65}} d^{3} x_{5}
$$

Similarly

$$
A_{\mu}^{b}(5)=\int J_{k+}^{b} \frac{\left(r_{6}, t_{5}-r_{56}\right)}{r_{56}} d^{3} x_{6}
$$

Thus $A_{\mu}^{a}$ and $A_{\mu}^{b}$ give the retarded fields emitted from the positive frequency parts of $J_{\mu}^{a}$ and $J_{\mu}^{b}$. It can be seen from the definition of the current,Equation (1I), in terms of eigenfunctions

$$
\begin{gathered}
J_{\mu}^{a}(s)=e^{2} \sum_{t E_{n}} \sum_{t E_{m}} \phi_{n}\left(x_{3}\right) \bar{\phi}_{n}\left(x_{s}\right) \gamma_{a \mu} \phi_{m}\left(x_{s}\right) \bar{\phi}_{m}\left(x_{1}\right) e^{-i E_{n} t_{3}+i E_{n} t_{1}-i\left(E_{m}-E_{n}\right) t_{5}} \\
\text { for } t_{3}>t_{s}>t_{1}
\end{gathered}
$$

that the positive frequency part of the current is the part 
produced by transitions which lose kinetic energy. The result is essentially independent of the manner in which the interaction term of (1) is split into two. Thus, if one interchanges the functions in the definitions of $A_{\mu}^{a}$ and $A_{\mu}^{b}$, Equations (8) and (10), one would find instead of (15), the advanced fields arising from the negative frequency parts of $J_{\mu}^{a}$ and $J_{\mu}^{p}$ respectively. However, an advanced field travelling outward from a particle which is gaining kinetic energy must be interpreted as a retarded. field travelling inward to be absorbed by the particle.

These results are valid also if one of the particles is a positron. Suppose for definiteness that particle a is a positron. According to Feynman's theory of positrons (16), it is described as an electron travelling backwards in time with negative kinetic energy. In this case the order in time of events in Equations (1) and (8) is $t_{3}<t_{5}<t_{1}$ and the current of a defined by Equation (11) is the sum over negative energy states:

$$
J_{\mu}^{a}(s)=e^{2} \sum_{-E_{n}} \sum_{-E_{m}} \phi_{n}\left(x_{3}\right) \bar{\phi}_{n}\left(x_{s}\right) \gamma_{a \mu} \phi_{m}\left(x_{5}\right) \bar{\phi}_{m}\left(x_{1}\right) e^{-i E_{n} t_{s}+i E_{m} t_{1}-i\left(E_{m}-E_{n}\right) t_{s}}
$$

Thus the positive frequency part of the current defined by Equation (14) is the part for which $\left|\mathrm{E}_{\mathrm{m}}\right|<\left|\mathrm{E}_{n}\right|$; and, since the order of events is reversed, this part corresponds to transitions $\left|E_{n}\right| \rightarrow\left|E_{m}\right|$ in which the positron loses kinetic energy.

The other three types of interactions can be worked out in a similar way. For the $\delta_{-}\left(\hat{S}_{s b}^{2}\right)$ photon kernel one finds

$$
\begin{aligned}
A_{\mu}^{a}(6) & =\frac{1}{2} \int \frac{\delta-\left(t_{56}-r_{56}\right)}{r_{56}} J_{\mu}^{a}(5) d^{4} x_{5} \\
& =\int \frac{J_{\mu}^{a}+\frac{\left(r_{5}, t_{6}+r_{56}\right)}{r 56} d^{3} x_{5} .}{} .
\end{aligned}
$$


and

$$
\begin{aligned}
A_{\mu}^{b}(5) & =\frac{1}{2} \int \frac{\delta_{-}\left(-t_{56}-r_{56}\right)}{r_{56}} J_{\mu}^{b}(6) d^{4} x_{6} \\
& =\int \frac{J_{k+}^{b}\left(r_{6}, t_{5}+r_{56}\right)}{r_{56}} d^{3} x_{6}
\end{aligned}
$$

which give the advanced fields emitted by $a$ and $b$ respectively. The $D_{+}$and $D_{-}$kernels can be split into two terms each, since

$$
\delta\left(t_{56}-r_{56}\right)=\frac{1}{2}\left\{\delta_{+}\left(t_{56}-r_{56}\right)+\delta_{-}\left(t_{56}-r_{56}\right)\right\}
$$

and the results given in Table I follow easily. These kernels correspond to the singular solutions of the Maxwell equations described by stueckelberg (3).

The difficulty mentioned by Wheeler and Feynman (25), that a retarded interaction of $a$ on $b$ corresponds to an advanced interaction of $b$ on $a$, is valid for the Stueckelberg type of interactions but not the purely retarded $\delta_{+}$-type of interaction. The classical derivation by Darwin (33) of the Hamiltonian for two charges was based implicitly on a Stueckelberg type of interaction and not the retarded interaction. However as will be shown for the quantum mechanical derivation of the Hamiltonian for two electrons it makes no difference up to the second order in $\mathrm{v} / \mathrm{c}$ which of the four possible interactions is assumed.

Let us consider the matrix elements of the interaction term in Equation ( 1 ) between the two-electron states

$$
\bar{g}_{a}\left(x_{3}\right) \bar{g}_{b}\left(x_{4}\right) e^{i\left(E_{a} t_{3}+E_{b} t_{4}\right)} \quad \text { and } \quad f_{a}\left(x_{1}\right) f_{b}\left(x_{2}\right) e^{-i\left(E_{a}^{0} t_{1}+E_{b}^{0} t_{2}\right)}
$$

which can be assumed for simplicity to be positive energy states. The volume integrals can be carried out by using the general relation 


$$
\begin{gathered}
-18- \\
\int K_{+}(2,1) f\left(x_{1}\right) e^{-i E t_{1}} d v_{1}=f\left(x_{2}\right) e^{-i E t_{2}}
\end{gathered}
$$

We then obtain for the matrix element

$$
M=-i e^{2} \iint \bar{g}_{a}\left(x_{s}\right) \bar{g}_{b}\left(x_{b}\right) \gamma_{a \mu} \gamma_{b \mu} \delta_{f}\left(s_{s b}^{2}\right) f_{a}\left(x_{s}\right) f_{b}\left(x_{b}\right) e^{i\left(E_{a}-E_{a}^{g}\right) t_{s}} e^{i\left(E_{b}-E_{b}^{0}\right) t_{b}} d^{4} x_{s} d^{4} x_{b}
$$

The time integrals $d t_{5}, d t_{6}$ extend over time $T$ which must be taken very long. Now if we consider only transitions which conserve energy then the integrand is proportional to the time difference $t_{56}$, except for small effects at the end points which vanish as $T$ becomes large. The integration over $t_{6}$ then gives a result proportional to the time. Thus

$$
M=-i e^{2} T \iint \bar{g}_{a}\left(x_{5}\right) \bar{g}_{b}\left(x_{6}\right) \gamma_{a \mu} \gamma_{b \mu} \delta_{+}\left(s_{56}^{2}\right) f_{a}\left(x_{5}\right) f_{b}\left(x_{6}\right) e^{-i\left(E_{a}-E_{a}^{2}\right) t_{56}} d^{3} x_{5} d^{3} x_{6} d t_{56},
$$

$$
\text { where } E_{a}-E_{a}^{0}=-\left(E_{b}-E_{b}^{0}\right) \text {. }
$$

Substituting for the photon kernel from Table $I$ and completing the time integration gives the result

$M=-i e^{2} T \iint d^{3} x_{5} d^{3} x_{b} \bar{g}_{4}\left(x_{5}\right) \bar{g}_{b}\left(x_{6}\right) \gamma_{a \mu} \gamma_{b \mu} f_{a}\left(x_{5}\right) f_{b}\left(x_{b}\right) r_{56}^{-1}\left\{\begin{array}{l}e^{i\left(E_{4}-E_{0}^{0}\right) r_{56} ; E_{a}>E_{a}^{0}} \\ e^{-i\left(E_{a}-E_{4}^{0}\right) r_{56}} ; E_{4}<E_{a}^{0}\end{array}\right.$. By using the relations

$$
\bar{g}=g^{*} \beta \text { and } \gamma_{\mu}=\beta \alpha_{\mu}
$$

this can be written as $M=-i V T$, where

$$
\begin{aligned}
V=e^{2} \iint d^{3} x_{5} d^{3} x_{b} g_{a}^{*}\left(x_{5}\right) g_{b}^{*}\left(x_{b}\right) \alpha_{a \mu} \alpha_{b \mu} f_{a}\left(x_{5}\right) f_{b}\left(x_{b}\right) \\
\quad \times r_{5 b}^{-1}\left[\cos \left(E_{a}-E_{a}^{0}\right) r_{5 b}+i \sin \left|E_{a}-E_{a}^{0}\right| r_{5 b}\right]
\end{aligned}
$$

The same expression can be obtained for electrons moving in a 
common external field, $\mathbb{E}$; it is only necessary to replace the free electron kernels appearing in Equation (1) by kernels for the propagation of electrons in the field. The analysis following Equation (1) remains valid.

As Feynman has pointed out, an amplitude M proportional to the time may be regarded as the first order term of the transformation $\exp (-i V t)$. In general $V$ is not hermitian, the antihermitian part arising from the sine factor in the integrand. The hermitian part can be expressed as the matrix element of an operator by expanding the cosine factor in a power series. This is the method used by. Bethe and Fermi (34). Up to the second order in $\mathrm{v} / \mathrm{c}$ the operator may be written

$$
e^{2}\left\{\frac{\alpha_{a \mu} \alpha_{b \mu}}{r_{a b}}-\left[H_{a},\left[\alpha_{a \mu} \alpha_{b \mu} r_{a b}, H_{b}\right]\right]=\frac{e^{2}}{r_{a b}}\left\{1-\frac{1}{2}\left(\alpha_{a}-\alpha_{b}\right)-\frac{1}{2} \frac{\left(\alpha_{a} \cdot r_{a b}\right)\left(\alpha_{b} \cdot r_{a b}\right)}{r_{a b}^{2}}\right\},\right.
$$

which is the well known Breit formula (29).

The antihermitian part of $\mathrm{V}$ apparently cannot be written as the matrix element of an operator because of the absolute value sign on the argument. The first term from the power series for the sine function vanishes by orthogonality; the second term gives an antihermitian part to $V$ which in $|\exp (-i V T)|^{2}$ produces an exponental decay with time. This is related to the fact that the total probability for elastic scattering is not in general unity. For zero external field the antihermitian part in fact vanishes. This is to be expected, because radiation processes for two electron scattering are of third order and higher. For non zero external field the antihermitian part of $V$, when 
diagnonalized by choice of correct zero order wave-functions, would give part of the decay probability per unit time of the stationary two-electron states. The remaining contribution comes from the self-energy of each electron individually; since, as Oppenheimer has shown (5), it is the imaginary part of the total self energy of atomic electrons which gives the half width of the spectral lines.

The advanced interaction gives a similar expression for $\mathrm{V}$ but with the sign in front of $\left|E_{a}-E_{a}^{o}\right|$ reversed. Thus the assumption that the interaction is half retarded plus half advanced leads to cancellation of the sine term in the expression for $V$, giving an hermitian matrix with real eigenvalues. This result is in agreement with the Wheeler-Feynman absorber theory (25) in which radiation effects are accounted for by the action of the absorber.

The interactions of the Stueckelberg type give expressions for $V$ without the absolute value sign on $\left(E_{a}-E_{a}^{0}\right)$. The sine term is therefore not symmetric in the particles and cannot be expressed as the matrix element of an operator which is symmetric. The cosine term is exactly the same as for the retarded and advanced interactions and gives the Breit formula. 
III. EVALUATION OF THE FREE ELECTRON SELF-ENERGY MATRIX

The notation introduced by Feynman will be used throughout this section and the next. According to Feynman (20) the selfenergy of the free electron can be written in momentum space as the single integral

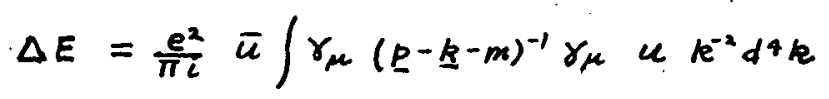

where $d^{4} k=(2 \pi)^{-2} d k_{4} d^{3} k, \quad(p-\underline{k}-m)^{-1}=(p-k+m)\left((p-k)^{2}-m^{2}\right)^{-1}$ and $u$ is a solution of the Dirac equation

$$
(R-m) u=0, \quad u^{*} u=1, \bar{u}=u^{*}(3
$$

The left hand side of (I) can therefore be written as $\Delta E u^{\prime} u$ or, since $E \Delta E=m \Delta m$ and $u^{n} u=\bar{u} \frac{E}{m} u$, as $\Delta m$ ūu. It is convenient to carry out the summation over $\mu$ in (1) right away by using

$$
\begin{array}{ll}
\gamma_{\mu} A \gamma_{\mu}=-2 \underline{ } & \text { for any } A=A_{\mu} \gamma_{\mu}, \\
\gamma_{\mu} \gamma_{\mu}=4 \quad, \underline{P} \mu=m u
\end{array}
$$

thus removing the $\gamma$-matrices and obtain

$$
\Delta m(\bar{u} u)=\frac{2 e^{2}}{\pi i} \bar{u}\left(m J_{1}+\underline{J}_{2}\right) u
$$

with

$$
J_{1 ; 2 \nu}=\int(1 ; k \nu)\left\{(p-k)^{2}-m^{2}\right\}^{-1} k^{-2} \cdot d^{4 k}
$$

It is now the integration over $k_{4}$ which exhibits fully the advantage of treating the problem in momentum space, as the distinctions between one-electron theory and hole theory and the 
different kinds of self-interaction can most converiently be made by assigning appropriate conventions regarding the positions of the poles of the integrals (5). As we are interested only in the case of the resting electron $(\vec{P}=0)$, we need calculate only $J_{1}$ and $J_{24}$ because $\vec{J}_{2}$ vanishes in this cas e by symmetry. If the integration over the variable $k_{4}$ is performed by completing the contour with a semicircle above the real $k_{4}$-axis the integrals (5) càn be written

$$
J_{1 ; 24}=2 i \int K^{2} d K \sum R_{1 ; 24}
$$

where $\Sigma$ has to be carried out over the respective residues at the poles positioned above the real $k_{4}$-axis. Each of the integrals (5) has four poles along the $k_{4}$-axis which are tabulated together with the corresponding residues of $J_{1}$ and $J_{24}$ in Table II.

TABLE II

Poles

$$
\text { Residues of } \mathrm{J}
$$

Residues of $\mathrm{J}$

$$
k_{4}^{\prime}=-K
$$$$
R_{1}^{\prime}=-\frac{E}{4 m^{2} K^{2}}
$$$$
R_{24}^{\prime}=\frac{E}{4 m^{2} K}
$$

$k_{4}^{\prime \prime}=k$

$$
R_{1}^{\prime \prime}=-\frac{E}{4 m^{2} R^{2}}
$$$$
R_{24}^{\prime \prime}=-\frac{E}{4 m^{2} K}
$$

$$
k_{4}^{\prime \prime \prime}=E-\sqrt{K^{2}+m^{2}}
$$$$
R_{1}^{\prime \prime \prime}=\frac{m^{2}+E \sqrt{k^{2}+m^{2}}}{4 m^{2} K^{2} \sqrt{k^{2}+m^{2}}}
$$$$
R_{24}^{\prime \prime \prime}=-\frac{E}{4 m^{2} \sqrt{k^{2}+m^{2}}}
$$$$
k_{4}^{\prime v}=E+\sqrt{k^{2}+m^{2}}
$$$$
R_{1}^{N=}=-\frac{\left(m^{2}-E \sqrt{K^{2}+m^{2}}\right)}{4 m^{2} K^{2} \sqrt{k^{2}+m^{2}}}
$$

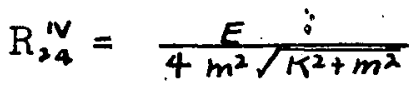


In the computation of this table extensive use has been made of the relation

$$
p_{4}^{2}=E^{2}=m^{2} .
$$

for the free rest electron.

The distinction between one-electron theory and hole theory is now made in the usual way by assigning for one-electron theory an infinitesimalipositive imaginary part to $\mathrm{E}$, thus bringing $\mathrm{k}_{4}^{\prime \prime \prime}$ and $k_{4}^{\prime \prime}$ above the real $k_{4}$-axis, while assignment of an infinitesimal negative imaginary part to $\mathrm{m}$ leads to the hole theory, bringing $k_{4}^{\prime \prime \prime}$ above and $k_{4}^{\prime \prime}$ below the real $k_{4}$-axis. There remain then four possibilities for locating the poles $k_{4}^{\prime}$ and $k_{4}^{\prime \prime}$ earch corresponding to a different kind of self-interaction as listed in Table $I$.

The photon kernels of main interest from the point of view of this thesis are the $\delta_{+}$and $\delta_{-}$functions which correspond to the purely retarded or purely advanced self-interactions. The interactions of the Stueckelberg type are included for the sake of completeness.

Thus the integration over $k_{4}$ in (5) as outlined above yields the following complete self-energy Table III for the free resting electron. 
$-24-$

TABLE III

Photon Kernel

$\delta_{+}$

$\delta$

$D_{+}$

D_ $\sum R$
$\Delta m$

One Electron Theory

$R^{\prime}+R^{\prime \prime}+R^{\prime v}$

$R^{\prime \prime}+R^{\prime \prime \prime}+R^{\prime \prime}$

$R^{m}+R^{\prime v}$

$R^{\prime}+R^{\prime \prime}+R^{\prime \prime \prime}+R^{\prime V}$

$$
\begin{aligned}
& \frac{e^{2}}{\pi m}\left\{E \int_{0}^{\infty} d k+\int_{0}^{\infty} k d k\right\} \\
& \frac{e^{2}}{\pi m}\left\{E \int_{0}^{\infty} d k-\int_{0}^{\infty} k d k\right\} \\
& \frac{2 e^{2}}{\pi m} E \int_{0}^{\infty} d k
\end{aligned}
$$

zero

Hole Theory

$\delta_{+} \quad R^{\prime}+R^{\prime \prime \prime}$

$\delta$

D.

D.
$R^{\prime \prime}+R^{\prime \prime \prime}$

$R^{\prime \prime \prime}$

$R^{\prime}+R^{n}+R^{\prime \prime \prime}$

$$
\begin{aligned}
& \frac{e^{2} m}{\pi} \int_{0}^{\infty} d K\left\{\frac{1}{\sqrt{K^{2}+m^{2}}}-\frac{K^{2}}{m^{2} \sqrt{K^{2}+m^{2}}}+\frac{K}{m^{2}}\right\} \\
& \frac{e^{2} m}{\pi} \int_{0}^{\infty} d K\left\{\frac{1}{\sqrt{K^{2}+m^{2}}}-\frac{K^{2}}{m^{2} \sqrt{K^{2}+m^{2}}}-\frac{K}{m^{2}}\right\} \\
& \frac{e^{2} m}{\pi} \int_{0}^{\infty} d K\left\{\frac{1}{\sqrt{K^{2}+m^{2}}}+\frac{E}{m^{2}}-\frac{K^{2}}{m^{2} \sqrt{K^{2}+m^{2}}}\right\} \\
& \frac{e^{2} m}{\pi} \int_{0}^{\infty} d K\left\{\frac{1}{\sqrt{K^{2}+m^{2}}}-\frac{E}{m^{2}}-\frac{K^{2}}{m^{2} \sqrt{K^{2}+m^{2}}}\right\}
\end{aligned}
$$

It can be seen that in the one-electron theory the assumpion that the interaction is half retarded plus half advanced, which corresponds to Dirac's assumption that the positive and negative energy photons be treated symmetrically, leads to cancollation of the quadratically divergent transverse part of the self energy. It is interesting to note that an interaction of the stueckelberg type $D$-, leads to vanishing self-energy in one-electron theory. Unfortunately this does not solve the 
self-energy problem, as in this case radiation corrections to scattering characterized by integrals of the type

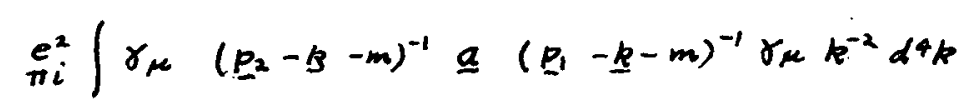

will vanish as well, because all poles of such integrals too are then located above the real $\mathrm{k}_{4}$-axis. This would contradict experimental evidence like the Lamb Shift and the anomalous magnetic moment of the electron. In the hole theory it is only the retarded interaction which makes the divergence logarithmic, all other interactions leading to quadratically divergent expressions for $\Delta \mathrm{m}$. Thus the assumption that the interaction is half retarded plus half advanced would remove the term $\mathrm{K} / \mathrm{m}^{2}$ in the integrand of $\Delta \mathrm{m}$ and destroy the logarithmic character of the divergence leaving a quadratically divergent expression for $\Delta \mathrm{m}$. 
IV. SELF ENERGY MATRIX FOR AN ELECTRON IN AN EXTERNAL FIELD

Feynman's kernel for the free electron interacting with itself may be generalized for an electron in an external field, E. by replacing the free electron kernel $K_{+}(2,1)$ by $K_{+}^{(A)}(2,1)$, the kernel in hole theory for the Dirac equation

$$
\left(i \nabla_{2}-A(2)-m\right) K_{+}^{(A)}(2,1)=i \delta(2,1)
$$

The kernel for an electron propagating in the external field and interacting with itself may then be written to first order in $e^{2}$ as

$$
K(2,1)=K_{4}^{(n)}(2,1)-i e^{2} \iint K_{t}^{(4)}(2,4) \gamma_{\mu} K_{t}^{(4)}(4,3) \gamma_{\mu} K_{+}^{(4)}(3,1) \delta_{+}\left(s_{43}^{2}\right) d^{4} x_{4} d^{4} x_{3}
$$

The second term arises because the electron may emit a photon at 3 and reabsorb it at 4 thus spending part of its time in an intermediate state. The wave function at 2 may be obtained in terms of the wave function at $I$ in the usual way according to the relation

$$
\psi(2)=\int K(2,1) \underline{N} \psi \psi(1) d V_{1},
$$

where $V_{1}$ is a hypersurface enclosing the point 2 and $\underline{N}$, is the inward drawn normal to $V_{1}$. Operating on the left with the Dirac operator ( $i \nabla_{2}-A(2)-m$ ) and using Equations (1) and (2) then gives the modified Dirac equation

$$
\left(i \underline{\nabla}_{2}-\underline{A}(2)-m\right) \psi(2)=e^{2} \int \gamma_{\mu} K_{+}^{(\mu)}(2,3) \gamma_{\mu} \psi(3) \delta_{+}\left(s_{23}^{2}\right) d^{3} x_{3} .
$$


This is similar in form to the equation given by Schwinger (31), but is obtained here by a relatively simple calculation.

The self-energy may be calculated as a perturbation by replacing $\psi$ by an eigenfunction, $\phi_{m}(x) \exp (-i E t)$, of the unperturbed Dirac equation. The self-energy is then the diagonal matrix element*

$$
W_{\dot{m}}=e^{2} \int \Phi_{m}\left(x_{2}\right) \gamma_{\mu} K_{t}^{(A)}(2,3) \gamma_{\mu} \phi_{m}\left(x_{3}\right) e^{-i E_{m} c_{23}} \delta_{+}\left(s_{23}^{2}\right) d t_{3} d^{3} x_{3} d^{3} x_{2} .
$$

This may be evaluated if the kernels are replaced by their Fourier representations

$$
\begin{aligned}
& \delta_{+}\left(s_{23}^{2}\right)=-\frac{1}{\pi} \int d 4 k e^{-i k x_{23} 3} k^{-2} \cdot \ldots \\
& K_{t}^{(A)}(x, 3)= \begin{cases}\sum_{t E_{n}} \phi_{n}\left(x_{2}\right) \bar{\phi}_{n}\left(x_{3}\right) e^{-i E_{n} t_{23}} ; t_{2}>t_{3} \\
\sum_{-E_{n}} \phi_{n}\left(x_{2}\right) \bar{\phi}_{n}\left(x_{3}\right) e^{-i E_{n} t_{23}} ; t_{2}<t_{3} .\end{cases}
\end{aligned}
$$

In one-electron theory $K_{+}^{(1)}(2,3)$ is replaced by the one-electron kernel $K_{0}^{(A)}(2,3)$ given by

$$
K_{0}^{(n)}(x, 3)=\left\{\begin{array}{cc}
\sum_{n} \phi_{n}\left(x_{2}\right) \bar{\phi}_{n}\left(x_{3}\right) e^{-i E_{n} t_{23}} & ; t_{2}>t_{3} \\
0 & ; t_{2}<t_{3} .
\end{array}\right.
$$

The distinction between the different types of interaction is now made as in Parts II and III by assigning different conventions for evaluating the poles of $\mathrm{k}^{-2}$ in the $\mathrm{k}_{4}$-complex plane.

- Provided either the unperturbed state $m$ is non degenerate or $W$ is diagonal by choice of correct zero order wave functions. 
The necessary splitting of the $t_{3}$ integration into two ranges $\left(-\infty, t_{2}\right)$ and $\left(t_{2}, \infty\right)$ gives rise to an imaginary part to the selfenergy according to the relation

$$
\frac{i}{\pi} \int_{0}^{\infty} e^{7 i \omega t} d t=i \delta_{ \pm}(\omega)=i \delta(\omega) \pm \frac{1}{\pi \omega}
$$

The integration over $\overrightarrow{\mathrm{K}}$ for the imaginary part can most conveniently be completed by making the dipole approximation and using the formula

$$
\int d^{3} K K^{-1} \delta(E-K)=\left\{\begin{array}{cc}
4 \pi E ; & E>0 \\
0 ; & E \leqslant 0 .
\end{array}\right.
$$

If we write $W m=\Delta \mathrm{E}_{m}-1 \Gamma_{\mathrm{m}} / 2$ then $\Gamma_{\mathrm{m}}$ produces in $|\exp (-1 W m t)|^{2}$ an exponentially decreasing amplitude with time; so $\Gamma_{\mathrm{m}}$ is just the decay probability per unit time for the state $m$. Finally the relations $\quad \delta=\phi^{x} \beta$ and $\gamma_{\mu}=\beta \alpha_{\mu}$

are used to simplify the expressions for $\Delta E_{m}$ and $\Gamma_{m}$ which are listed in Table IV. 
TABLE IV

\section{Photon}

Kernel $\Delta E_{m}$

$\Gamma_{m}$

\begin{tabular}{|c|c|c|}
\hline \multicolumn{3}{|c|}{ One-Electron Theory } \\
\hline$\delta_{t}$ & $\frac{e^{x}}{4 \pi^{2}} \int_{K}^{d^{3} K} \sum_{E_{n}} \frac{|V|^{2}}{E_{n}-E_{m}+K}$ & $\because \quad-2 e^{2} \sum_{E_{n}<E_{m}}|V|^{2}\left(E_{m_{2}}-E_{n}\right)$ \\
\hline$\delta_{-}$ & $-\frac{e^{2}}{4 \pi^{2}} \int d^{3} K K \sum_{E_{n}} \frac{|V|^{2}}{E_{n}-E_{m}-K}$ & $+2 e^{2} \sum_{0<E_{m}<E_{m}}|V|^{2}\left(E_{n}-E_{m}\right)$ \\
\hline$D_{+}$ & $\frac{e^{2}}{4 \pi^{2}} \int d^{3} K \sum_{E_{n}}\left\{\frac{|V|^{2}}{E_{n}-E_{m}+K} \quad \frac{|V|^{2}}{E_{n}-E_{m}-K}\right\}$ & $2 e^{2}\left\{\sum_{0<E_{m}<E_{n}}|V|^{2}\left(E_{n}-E_{m}\right)-\sum_{E_{n}<E_{m}}|V|^{2}\left(E_{m}-E_{n}\right)\right\}$ \\
\hline D_ & Zero & zero \\
\hline
\end{tabular}

Hole Theory
$\delta_{+} \mid \frac{e^{2}}{4 \pi^{2}} \int_{K}^{d^{3} K}\left\{\sum_{+} \frac{|V|^{2}}{E_{n}-E_{m}+K}\right.$
$\left.+\sum \frac{|V|^{2}}{E_{n}-E_{m}-K}\right\}$
$-2 e^{2} \sum_{0 \leqslant E_{n}<E_{m}} \mid V V^{2}\left(E_{m}-E_{n}\right)$
d. $-\left.\frac{e^{2}}{4 \pi^{2}}\right|_{K} ^{d^{3} K}\left\{\sum_{+} \frac{|V|^{2}}{E_{n}-E_{m}-K}+\sum \frac{|V|^{2}}{E_{n}-E_{m}+K}\right\}$
$\lambda e^{2}\left\{\left.\sum_{0<E_{m}<E_{n}}\left|V E^{2}\left(E_{m}-E_{m}\right)-\sum_{E_{n}<0<E_{m}}\right| V\right|^{2}\left(E_{m}-E_{n}\right)\right.$
$D_{+} \quad \frac{e^{2}}{4 \pi^{2}} \int_{K}^{d^{3} K} \sum_{+}\left\{\frac{|V|^{2}}{E_{n}-E_{m}+K}-\frac{|V|^{2}}{E_{n}-E_{m}-K}\right\}$
$2 e^{2} \sum_{+}|V|^{2}\left(E_{m}-E_{n}\right)$
D. $-\frac{e^{2}}{4 n^{2}} \int_{K}^{d^{3} K} \sum\left\{\frac{|V|^{2}}{E_{n}-E_{m}+K}-\frac{|V|^{2}}{E_{m}-E_{m}-K}\right\}$
$2 e^{2} \sum|V|^{2}\left(E_{m}-E_{m}\right)$

$V=V_{m n}^{\mu}=\int \phi_{m}^{n}(x) \alpha_{\mu} e^{-i \vec{\mu} \cdot \vec{r}} \phi_{n}(x) d^{3} x ; \quad \alpha_{\mu} \alpha_{\mu}=1-\vec{\alpha} \cdot \vec{\alpha}$ 
An inspection of Column 3 shows that only the $\delta_{+}$-interaction in the hole theory gives the correct rate of radiation from an excited state, as shown below. For simplicity this table is restricted to the case in which Em is positive. : In the oneelectron theory the $\delta_{+}$-interaction allows the electron to fall into the negative energy states with emission of a positive energy photon and the $\delta_{-}$-interaction allows transitions upward with emission of a negative energy photon. The $D_{+}$-interaction gives the same result as the $\left(\delta_{+}+\delta_{-}\right)$-interaction. The $D_{-}$-interaction gives a vanishing self-energy as for the radiation corrections to scattering referred to in section III. In the hole theory the $\delta_{-}-$ interaction contains a second set of terms allowing transitions to negative energy states. These terms must be associated with the possibility of pair creation out of the vacuum along with emission of a negative energy photon, but they will not be discussed further here. The stueckelberg interactions allow transitions both up and down to occur.

It appears that the longitudinal and scalar photons as well as the transverse photons contribute to $\Gamma$, but the first two contributions exactly cancel. For proof, the matrix element can be written in momentum space as

$$
V_{m n}^{\mu}=\int \phi_{m}^{*}(\vec{P}-\vec{k}) \phi_{n}(\vec{P})\left(\bar{u}_{m}(\vec{P}-\vec{k}) \gamma_{\mu} u_{n}(\vec{P})\right) d^{3} P,
$$

in which $u_{n}(p)$ is the Dirac spinor and $\phi_{n}(p)$ is the coefficient in the expansion of $\phi_{x}(x)$ in momentum eigenfunctions. It follows from the Dirac equation and its conjugate that

$$
k_{\bar{m}} V_{m n}^{\mu}=0
$$


For a free photon $k_{4}=-K$ so $V_{m i n}^{4}=V_{m n}^{K}$. Thus

$$
-\left|v_{m n}^{\kappa}\right|^{2}=\left|\vec{v}_{m n}\right|^{2}-\left|v_{m n}^{k}\right|^{2},
$$

which is just the sum over the transverse photons. Finally, averaging over all directions $\vec{K}$ and making the usual nonrelativistic approximations leads to the radiation formula given by Heitler (35). For a second proof see Feynman (20). 
V. THE ANOMALOUS MAGNETIC MOMENT OF THE ELECTRON

Luttinger. (32) has shown that the anomalous magnetic moment may be obtained from the self-energy of the electron in a uniform magnetic field by taking advantage of the existence of a particular unperturbed non-degenerate state for which $E_{m}=m$. For this state the contribution to $\Delta \mathrm{E}$ due to the change in mass of the electron is just $\Delta \mathrm{m}$ so that the mass renormalization requires only the direct subtraction of $\Delta \mathrm{m}$. The method of Luttinger is followed in calculating the anomalous magnetic moment predicted by the self-energy formulas given in Column 2 of Table IV. However the factor $|V|^{2}$ contains the sum over all four polarization directions, $\mu$, thus including the Coulomb interaction directly as the effect of interaction with longitudinal photons. This simplifies somewhat the calculation of the matrix elements which is given in Appendix $A$. The resulting expression for $\Delta \mathrm{E}$ is expanded in powers of the magnetic field strength $\mathrm{H}_{0}$. up to the first. The term independent of $H_{0}$ in each case is just the corresponding expression for $\Delta m$ from Table III (with $E_{m}=m$ ).

It was found that the renormalized self-energies could be expressed in terms of a single divergent integral $\mathrm{F}(\mathrm{m}, \mathrm{K})$ :

$$
\begin{aligned}
F(m, K)=\frac{e^{2}}{\pi} \frac{e H_{0}}{m} \int_{0}^{\infty} m d K & \left\{\frac{1}{2} \frac{m K^{2}-K^{3}}{\left(m^{2}+K^{2}\right)^{3 / 2}}+\frac{1}{6} \frac{5 K-m}{\left(m^{2}+K^{2}\right)^{3 / 2}}+\frac{K^{2}}{12 m\left(m^{2}+K^{2}\right)^{3 / 2}}\right. \\
& \left.+\frac{1}{6 m^{3}}\left(m-\sqrt{m^{2}+K^{2}}-3 m^{2}\right)\right\}
\end{aligned}
$$

The results are listed in Table $\mathrm{V}$. 


\section{TABLE V}

Photon Kernel

$\Delta E-\Delta m$

$\Delta E-\Delta m$

\begin{tabular}{ccc}
\hline & One-Electron Theory & \\
$\delta_{+}$ & $F(m, K)+F(-m,-K)$ & Quadratically divergent \\
$\delta_{-}$ & $F(m,-K)+F(-m, K)$ & Quadratically divergent \\
$D_{+}$ & $\delta_{+}+\delta-$ & Quadratically divergent \\
$D-$ & Zero & Zero \\
\hline & Hole Theory & \\
$\delta_{+}$ & $F(m, K)-F(-m, K)$ & Quadratically divergent \\
$\delta_{-}$ & $F(m,-K)-F(-m,-K)$ & Quadratically divergent \\
$D-$ & $F(m, K)+F(m,-K)$ & Quadratically divergent \\
$D-$ & $F(-m,-K)+F(-m, K)$ & \\
\hline
\end{tabular}


Table $V$ shows that the correct expression for the anomalous magnetic moment given by Luttinger and Schwinger is obtained only from the $\delta_{+}$-interaction in hole theory. The renormalized self-energy for this interaction is in ordinary units

$$
-\frac{1}{2 \pi} \frac{e^{2}}{\hbar c} \frac{e \hbar}{2 m c} H_{0} \text {, }
$$

which can be interpreted as an addition to the magnetic moment of $\alpha / 2 \pi$ Bohr magnetons. The remaining six non-vanishing expressions lead to a quadratically divergent anomalous magnetic moment.

These results show conclusively that there is no solution to the self-energy problem possible by introducing advanced selfinteractions or the corresponding negative energy virtual photons of Dirac. It must be assumed that the electron interacts with itself via its retarded field. The one-electron theory must be rejected for the same reasons. 
VI. THE RETARDED FIELD OF A FREE REST ELECTRON

In this section the virtual field of a free rest electron is investigated for the purpose of determining the character of the divergence of the field strengths and of the total energy of the field both in one-electron theory and in hole theory. 'Since the results of the previous section indicate that only the retarded field of the electron has a physical meaning this field only will be calculated. This means using the conventional quantum electrodynamics with positive energy photons.

The following results from perturbation theory wili be required. If $\psi_{m}$ is a stationary state solution of the Schrodinger equation

$$
H \psi_{m}=W_{m} \psi_{m},
$$

where $H$ can be written as $H_{0}$ plus a small perturbation $\lambda V$ (with $\lambda$ later put equal to unity), then $\psi_{m}$ can be expressed in terms of the eigenfunctions $\psi_{m}^{\circ}$ of the unperturbed Hamiltonian $H_{0}$ up to second order in $\lambda$ by

$$
\begin{aligned}
& \psi_{m}=\psi_{m}^{0}+\lambda \sum_{k}^{\prime} \frac{V_{K m}}{W_{m}^{0}-W_{k}^{0}} \dot{\psi}_{k}^{0}+\lambda^{2} \sum_{\kappa}^{0} \sum_{n}^{\prime} \frac{V_{K n} V_{n m}}{\left(W_{m}^{0}-W_{r}^{0}\right)\left(W_{m}^{0}-W_{n}^{0}\right)} \psi_{k}^{0} \\
& -\lambda^{2} \sum_{k}^{\prime} \frac{V_{k m} V_{m m}}{\left(W_{m}^{0}-W_{k}^{0}\right)^{2}} \psi_{k}^{0}-\frac{1}{2} \lambda^{2} \sum_{k}^{\prime} \frac{\left|V_{n m}\right|^{2}}{\left(W_{m}^{0}-W_{k}^{0}\right)^{2}} \psi_{m}^{0},
\end{aligned}
$$

where $\quad H_{0} \psi_{n}^{0}=W_{n}^{0} \psi_{n}^{0}, \quad V_{k n}=\left(\psi_{n}^{0}, V \psi_{n}^{0}\right), \sum_{n}^{\prime}=\sum_{n \neq m}$.

The expectation value of any Hermitian operator A may then be written up to second order as 


$$
\begin{aligned}
& \bar{A}=\left(\psi_{m}, A \psi_{m}\right)=\left(\psi_{m}^{0} A \psi_{m}^{0}\right)+\lambda\left\{\sum_{\kappa}^{0} \frac{V_{k m}}{W_{m}^{0}-W_{k}^{0}}\left(\psi_{m}^{0}, A \psi_{k}^{0}\right)+\text { conj. }\right\} \\
& +\lambda^{2} \sum_{K}^{1} \sum_{n}^{1} \frac{V_{n m}^{*}}{W_{n}^{0}-W_{n}^{0}} \frac{V_{n m}}{W_{m}^{0}-W_{n}^{0}}\left(\psi_{n}^{0}, A \psi_{n}^{0}\right) \\
& \dot{+} \lambda^{2}\left\{\sum_{\kappa}^{\prime} \sum_{n}^{\prime} \frac{V_{n n}}{W_{n}^{0}-W_{n}^{0}} \frac{V_{n m}}{W_{m}^{0}-W_{n}^{0}}\left(\psi_{m}^{0}, A \psi_{n}^{0}\right)+\text { conj. }\right\} \\
& -\lambda^{2}\left\{\sum_{k}^{\prime} \frac{V_{k m} V_{m m}}{\left(W_{m}^{0}-W_{k}^{0}\right)^{2}}\left(W_{m}^{0}, A W_{k}^{0}\right)+\text { conj. }\right\}-\lambda^{2} \sum_{k}^{\prime} \frac{\left|V_{k m}\right|^{2}}{\left(W_{m}^{0}-W_{k}^{0}\right)^{2}}\left(W_{m,}^{0}, A W_{m}^{0}\right) .
\end{aligned}
$$

For the interaction of a single electron with the electromagnetic field

$$
\begin{gathered}
V=-e \overrightarrow{\alpha \cdot \vec{A}}, \\
\vec{A}=L^{-3 / 2} \sum_{k} \sum_{\gamma=1}^{2} q_{k, \gamma} \vec{e}_{k, \gamma} e^{i k \cdot r}+q_{k, \gamma}^{n} \vec{e}_{k, \gamma} e^{-i k, r},
\end{gathered}
$$

and the operators $q_{n, y}$ have the matrix elements

$$
q_{n, n+1}=q_{n+1, n}^{x}=\sqrt{\frac{h c}{k}(n+1)}
$$

A. NUMBER OF PHOTONS IN THE VIRTUAL FIELD

The operator of photon number is given according to (5) by

$$
N_{k, \gamma}=\frac{k}{h c} q_{k, \gamma}^{*} q_{k, \gamma}
$$

and the average number of photons is given by

$$
\bar{N}=\sum_{k, \gamma}\left(\psi_{m}^{*}, N_{k, \gamma} \cdot \psi_{m}\right)
$$

where $\mathrm{m}$ is the state which in zero order approximation contains no photons and either one electron or one electron plus the Dirac 
sea. When this expression is substituted into equation (3) the only non-vanishing terms are

$$
\bar{N}=\lambda^{2} \sum_{k, \gamma} \sum_{n}^{\prime} \frac{\left|V_{n m}\right|^{2}}{\left(W_{m}^{0}-W_{n}^{0}\right)^{2}}\left(\psi_{n}^{0}, N_{m, r} \psi_{n}^{0}\right)
$$

This expression is evaluated in Appendix B. In one-electron theory the result is

$$
\vec{N}=\frac{1}{2 \pi} \frac{e^{2}}{\hbar c}\left(\frac{\hbar}{m c}\right)^{2} \int_{0}^{\infty} k d k,
$$

which is quadratically divergent. The energy of the field is therefore cubically divergent. This divergence is partly compensated, as Weisskopf (8) has shown, by the kinetic energy of the electron in the intermediate states leaving a quadratically divergent self-energy for the electron. In hole theory the value of $\overline{\mathrm{N}}$ relative to the vacuum is zero. Thus, in hole theory the self-energy is just the kinetic energy of the electron in the intermediate states which is only logarithmically divergent. 
B. THE ELECTRIC AND MAGNETIC FIELDS OF A REST ELECTRON

The fields about a Dirac electron at rest may be described in terms of a correlation function which gives the products, averaged over the electron coordinates, of the probability density for the electron at $\vec{r}$ and the field intensity at $(\vec{r}+\vec{R})$. This method is an extension of the method used by Weisskopf for the charge and dipole densities in the neighbourhood of an electron.

(a). The Transverse Field

The transverse field strengths at a distrance $\vec{R}$ from an electron are defined in accordance with the idea given above, as the expectation values:

$$
\begin{aligned}
& \vec{E}_{\perp}(\vec{R})=\int \psi^{*}(\vec{r}, q) \vec{E}_{1 \text { op }}(\vec{r}+\vec{R}) \psi(\vec{r}, q) d \vec{r} d q, \\
& \vec{H}(\vec{R})=\int \psi^{*}(\vec{r}, q) \vec{H}_{\text {op }}(\vec{r}+\vec{R}) \psi(r, q) d \vec{r} d q,
\end{aligned}
$$

The integration is to be carried out over all coordinates $q$ of the field oscillators and over the coordinates $\vec{r}$ of the electron. The operators for the field strengths are obtained from equation (4):

$$
\begin{aligned}
& \vec{E}_{10 p}(\vec{r})=\frac{1}{c} \dot{A}(\vec{r})=\frac{l}{L^{3 / 2}} \sum_{k, r} q_{k, \gamma} \vec{e}_{k, \gamma} k e^{i k_{1} r}-q_{k, \gamma}^{x} \vec{e}_{k r} k e^{-i k_{1} r}, \\
& \vec{H}_{o p}(\vec{r})=\vec{\nabla} \times \vec{A}(\vec{r})=\frac{i}{L^{3 / 2}} \sum_{k, \gamma} q_{k, \gamma}\left(\vec{k} \times \vec{e}_{k, \gamma}\right) e^{i k, r}-q_{k, \gamma}\left(\vec{k} \times \vec{e}_{k, \gamma}\right) e^{-i k_{1} r} .
\end{aligned}
$$

The field strengths defined by (11) and (12) may now be evaluated for a rest electron with spin in the positive $z$ 
direction, using equations (3) to (6). (See Appendix C). The results are:

(i) one-electron theory:

$$
\begin{aligned}
& \vec{E}_{1}(\vec{R})=0 \\
& \vec{H}(\vec{R})=\frac{1}{R^{3}}\left\{2\left(\vec{M} \cdot \vec{R}_{1}\right) \vec{R}_{1}+\left[\vec{R}_{1} \times\left[\vec{R}_{1} \times \vec{M}\right]\right]\right.
\end{aligned}
$$

where $\vec{R}$, is a unit vector in the direction $\vec{R}$ and $\vec{M}$ is the electron dipole moment e $\hbar / 2 m c$. These are just the fields associated with a magnetic dipole of strength $M$.

(ii) hole theory:

$$
\begin{aligned}
\vec{E}_{l}(\vec{R})= & , \\
\vec{H} \cdot(\vec{R})= & \frac{1}{R^{3}} \frac{2}{\pi} \frac{R}{\lambda_{0}}\left\{\int_{0}^{1} d x K_{0}\left(\frac{R}{\lambda_{0}} x\right)-K_{0}\left(\frac{R}{\lambda_{0}}\right)\right\}\left\{2\left(\vec{M} \cdot \vec{R}_{1}\right) \vec{R}_{1}+\left[\overrightarrow{R_{1}} \times\left[\vec{R}_{1} \times \vec{M}\right]\right]\right\} \\
& +\frac{1}{R^{3}} \frac{2}{\pi}\left(\frac{R}{\lambda_{0}}\right)^{2} K_{1}\left(\frac{R}{\lambda_{0}}\right)\left\{\vec{M}-\left(\vec{M} \cdot \overrightarrow{R_{1}}\right) \overrightarrow{R_{1}}\right\},
\end{aligned}
$$

where $\lambda_{0}$ is the compton wavelength $\hbar / m c$ and $K_{\nu}$ is the Hankel function represented by

$$
K_{\nu}(z)=\int_{0}^{\infty} e^{-z \cosh t} \cosh \nu t d t .
$$

The asymptotic values of $\vec{H}$ for small $R$ and large $R$ can be easily worked out with this representation. For large $z$

$$
\begin{aligned}
& K_{\nu}(z) \longrightarrow e^{-z} \int_{0}^{\infty} e^{-z^{2} \frac{t^{2}}{2}} d t=\left(\frac{\pi}{2}\right)^{\frac{1}{2}} \frac{e^{-z}}{z^{\frac{1}{2}}}, \\
& \int_{0}^{\infty} d x K_{0}(x z)=\frac{1}{z} \int_{0}^{\infty} d t \frac{1-e^{-z \cosh t}}{\cosh t} \rightarrow \frac{\pi}{2} \frac{1}{z}-\left(\frac{\pi}{2}\right)^{\frac{1}{2}} \frac{e^{-z}}{z^{3 / 2}} .
\end{aligned}
$$

For small z

$$
\begin{aligned}
& K_{0}(z) \rightarrow \int_{0}^{\infty} \frac{d t}{1+z \cosh t}=-\log z, \quad \int_{0}^{1} d x K_{0}(x z) \rightarrow 1-\log z, \\
& K_{1}(z)=-\frac{d}{d z} K_{v}(z) \rightarrow \frac{1}{z} .
\end{aligned}
$$


Thus $\vec{H}$ has the asymptotic forms

$$
\begin{aligned}
\vec{H}(\vec{R}) \rightarrow & { }_{R^{3}}^{\prime}\left\{1-\left(\frac{2}{\pi}\right)^{\frac{1}{2}}\left(\frac{R}{\lambda_{0}}\right)^{\frac{1}{2}} e^{-R / \lambda_{0}}\right\}\left\{2\left(\vec{M} \cdot \vec{R}_{1}\right) \vec{R}_{1}+\left[\vec{R}_{1} \times\left[\vec{R}_{1} \times \vec{M}\right]\right]\right\} \\
& +\frac{1}{R^{3}}\left(\frac{2}{\pi}\right)^{\frac{1}{2}} \cdot\left(\frac{R}{\lambda_{0}}\right)^{3 / 2} e^{-R / \lambda_{0}}\left\{\vec{M}-\left(\vec{M} \cdot \vec{R}_{1}\right) \vec{R}_{1}\right\} ; R \gg \lambda_{0} \\
\vec{H}(\vec{R}) \rightarrow & \frac{2}{\pi} \frac{1}{\lambda_{0}} \frac{1}{R^{2}}\left\{\vec{M}-\left(\vec{M} \cdot \vec{R}_{1}\right) \vec{R}_{1}\right\} ; R<\lambda_{0} .
\end{aligned}
$$

For large values of $R$ the magnetic field goes over into the value found in one-electron theory. For small $R$ the singularity in the field is only $\mathrm{R}^{-2}$ compared to $\mathrm{R}^{-3}$ for one-electron theory. This form of the field in hole theory results from the polarization of the vacuum in the neighbourhood of the electron. The mean radius of the polarization is of the order of the Compton wavelength $\lambda_{b}$.

(b) The Coulomb Field

The Coulomb field is móst easily calculated by using second quantization for the electrons. The operator for the Coulomb field is defined by the correlation function

$$
\vec{E}_{11}(R)=-\vec{\nabla} \int \rho(\vec{r}) \phi_{o p}(\vec{r}+\vec{R}) d \vec{r} ;
$$

where

$$
\phi_{o p}(\vec{r})=e \int \frac{\rho\left(r^{\prime}\right)}{\left|\vec{r}^{\prime} \vec{r}^{\prime}\right|} d \vec{r}^{\prime}
$$

and

$$
\rho(r)=\sum_{j, k} a_{j}^{\alpha} a_{k}\left(u_{j}^{*} u_{k}\right) e^{i(k-\jmath) \cdot r} L^{-3},
$$


where the $u_{k}$ are Dirac spinors and $a_{k}$ and $a_{k}$ are the operators for creation and annibilation respectively of an electron in the state $K$. The operators satisfy, the anticommutation relations

$$
a_{k}^{*} a_{j}+a_{j} a_{k}^{*}=\delta_{k j} .
$$

The Coulomb field is given to first order in e by the expectation value of $\overrightarrow{\mathrm{E}}_{11}(R)$ in the unperturbed state. When this is evaluated with the help of the anticommutation relations (22) (See Appendix D), the results are:

(i) one-electron theory

$$
\vec{E}_{u}(R)=-\vec{\nabla} \frac{e}{R}
$$

(ii) hole theory

$$
\vec{E}_{1 \prime}(R)=-\vec{\nabla} \frac{2}{\pi} \frac{e}{\lambda_{0}} \int_{0}^{1} d x K_{0}\left(\frac{R x}{\lambda_{0}}\right),
$$

which has the asymptotic values

$$
\begin{aligned}
& \vec{E}_{11}(R) \rightarrow-\vec{\nabla}\left\{\frac{e}{R}-\frac{e}{R}\left(\frac{2}{\pi}\right)^{\frac{1}{2}} e^{-R / \lambda_{0}}\left(\frac{R}{\lambda_{0}}\right)^{-3 / 2} ; R \gg \lambda_{0},\right. \\
& \vec{E}_{1,}(R) \rightarrow-\vec{\nabla} \frac{2}{\pi} \frac{e}{\lambda_{0}}\left\{1-\log \frac{R}{\lambda_{0}}\right\} \quad ; R \ll \lambda_{0} .
\end{aligned}
$$

The result obtained for one-electron theory shows that the electron must be treated as a point charge. In hole theory however the polarization of the vacuum reduces the singularity of the potential to logarithmic. It is interesting to note that the charge density of a single electron in the vacuum found by Weisskopf (8) 


$$
\begin{aligned}
& -42- \\
& \operatorname{eg}(r)=\frac{e}{(2 \pi)^{3}} \frac{1}{\lambda_{0}^{3}} \int d^{3} k\left(1+k^{2}\right)^{-\frac{1}{4}} e^{-i \frac{k_{0}^{*} r_{0}^{2}}{\lambda_{0}}},
\end{aligned}
$$

which has the asymptotic values

$$
\begin{array}{cc}
\frac{3}{8 \pi} \cos \frac{\pi}{8} \frac{e}{\lambda_{0} / 4} r^{-11 / 4} e^{-r \lambda_{0}} \int_{0}^{\infty} d y \sqrt{y} e^{-y / 2} ; r \gg \lambda_{0}, \\
\frac{e}{\lambda_{0}^{1 / 2}} \frac{1}{\pi^{3 / 2}}(2 r)^{-5 / 2} & ; r \ll \lambda_{0},
\end{array}
$$

gives the above Coulomb field (24) if this density is substituted into the definition (19). A similar relation should hold for the magnetic field in hole theory, since the dipole density is also given by (26), but the calculation is involved and has not been done here. 
$-43-$

VII. APPENDIX

A. THE ANOMALOUS MAGNETIC MOMENT

The matrix elements $V_{n, m}^{\mu}$ will be evaluated first. An addition subscript, $i$, is used to label the four classes of eigenfunction $\psi_{s, i}$ which are listed by Luttinger; the double subscript $s, i$ stands for the single subscript $\dot{n}$ used by tuttinge.

$$
V_{s i m}^{4}=\int d^{3} x \psi_{s, L}^{*} \psi_{m} e^{-i \kappa \cdot r}=
$$

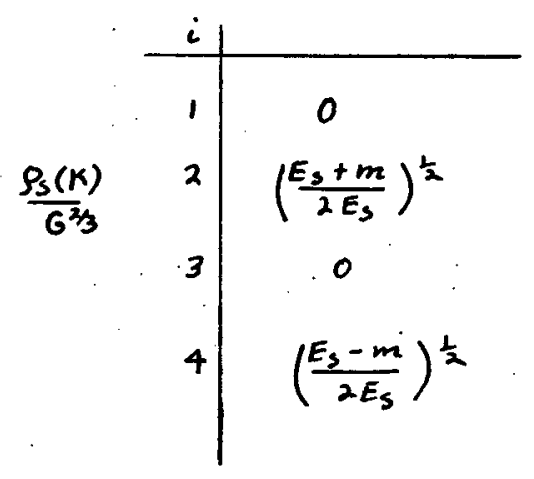

where

$$
\begin{aligned}
& P_{s}(K)=G^{2 / 3} \delta_{K_{2} p_{2}} \delta_{K_{3} P_{3}} \frac{(-1)^{s}}{2^{s / 2}(s !)^{\frac{1}{2}}}\left(\frac{K_{3}+c K_{1}}{\left(e H_{0}\right)^{\frac{2}{2}}}\right)^{S} \exp \left[-\frac{K_{2}^{2}+K_{1}^{2}+2 c K_{2} K_{1}}{4 e H_{0}}\right], \\
& E_{s}=+\left[m^{2}+P_{3}^{2}+2 e H_{0} s\right]^{\frac{1}{2}}
\end{aligned}
$$

$$
\left|\rho_{\delta}(K)\right|^{2}=\epsilon^{4 / 3} \delta_{K_{2} p_{2}} \delta_{K_{2} P_{3}} \frac{\eta^{s}}{s !} e^{-\eta} ; \eta=\frac{r^{2}}{2 e H_{0}}, \mu^{2}=K_{1}^{2}+K_{2}^{2}
$$

$$
\left|\vec{V}_{s, c ; m}\right|^{2}=\left.1 \int d^{3} x \psi_{s, l}^{N} \vec{\alpha} \psi_{m} e^{-i k \cdot r}\right|^{2}=2\left|V_{s_{L j} m}^{\prime}\right|^{2}+\left|V_{s c ; m}^{3}\right|^{2}
$$

because $\quad \alpha_{1} \psi_{m}=-i \alpha_{2} \psi_{m}$ 


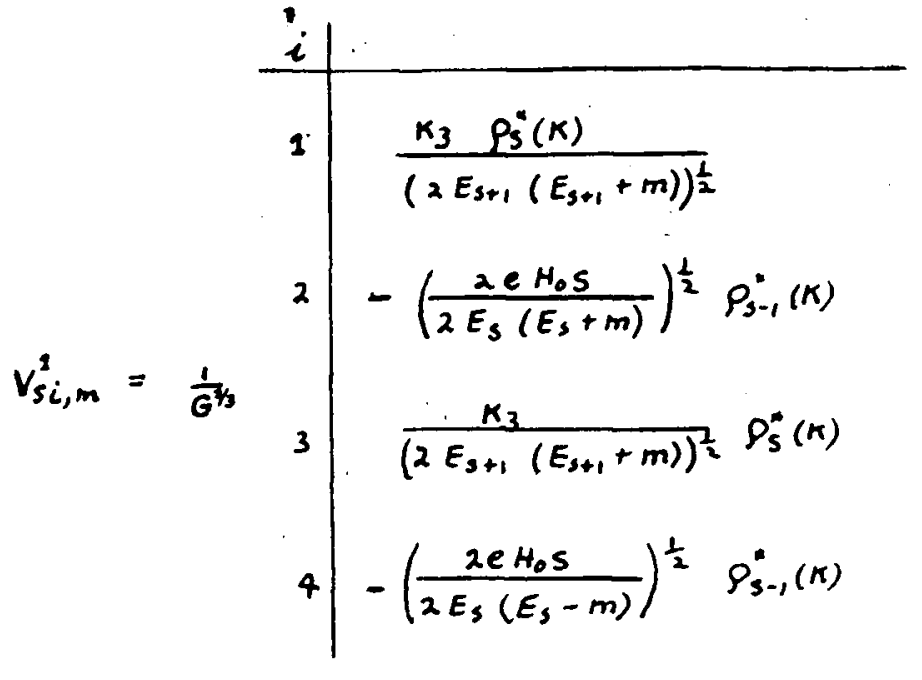

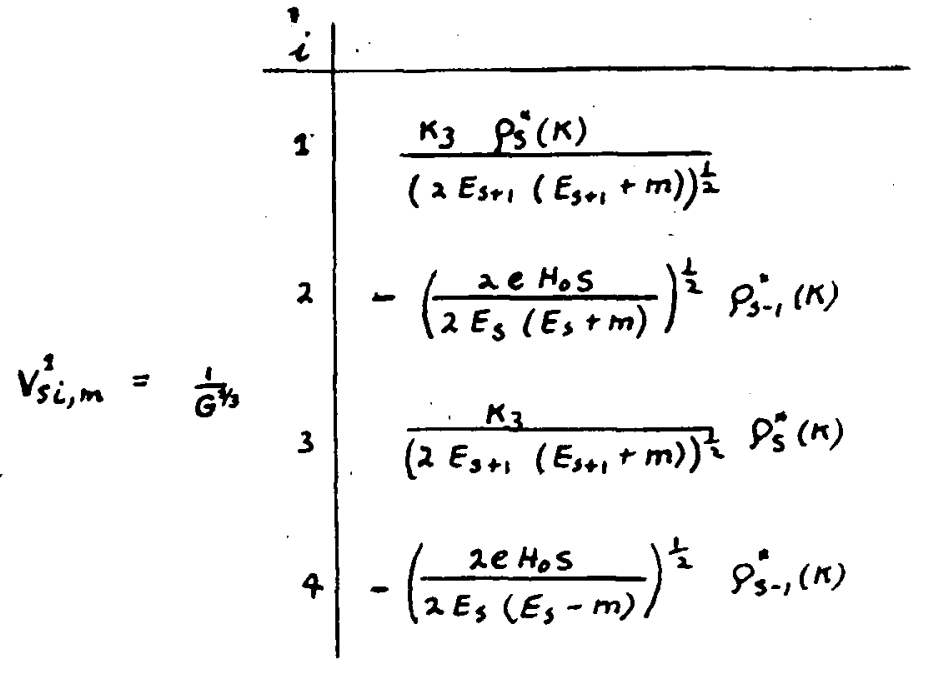

$$
\begin{aligned}
& V_{s i, m}^{3}=\frac{1}{G^{2 / 3}} \\
& 1 \quad\left(\frac{2 e H_{0}(s+1)}{2 E_{s+1}\left(E_{s+1}+m\right)}\right)^{\frac{1}{2}} \rho_{s+1}^{\prime}(k) \\
& 2 \frac{K_{3}}{\left(2 E_{s}\left(E_{s}+m\right)\right)^{\frac{1}{2}}} \rho_{s}^{\prime}(k) \\
& 3 \quad\left(\frac{2 \cdot e H_{0}(s+1)}{2 E_{s+1}\left(E_{s+1}-m\right)}\right)^{\frac{1}{2}} \rho_{2+1}^{\prime \prime}(k) \\
& 4 \frac{k_{3}}{\left(2 E_{s}\left(E_{s}-m\right)\right)^{\frac{1}{2}}} \rho_{s}^{\prime}(k) \\
& \left|V_{s i, m}^{\mu}\right|^{2}=\left|V_{s L, m}^{4}\right|^{2}-\left|\vec{v}_{s i, m}\right|^{2}
\end{aligned}
$$

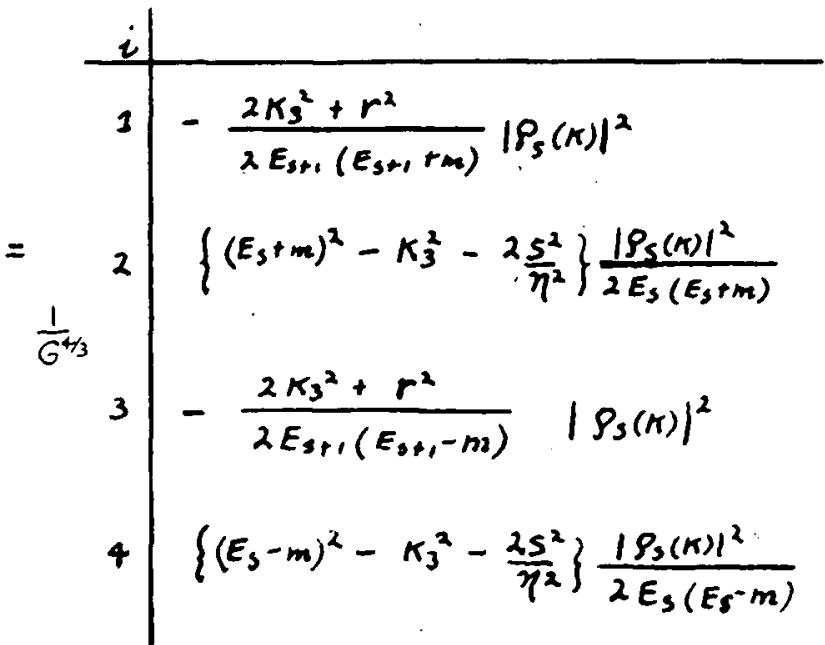$$
-44=
$$

(6) 
In simplifying Equation (7) frequent use has been made of the Equations (3). For example

$$
2 \text { e } H_{0}(s+1)\left|\rho_{s+1}\right|^{2}=\frac{r^{2}}{\eta^{2}}(s+1)\left|\rho_{s+1}\right|^{2}=r^{2}\left|\rho_{s}\right|^{2}
$$

The self-energies listed in Table IV can now all be expressed in terms of a simple function $F(m, k)$ :

$$
\begin{aligned}
& \frac{e^{2}}{4 \pi^{2}} \sum_{+} \int \frac{d^{3} K}{K} \frac{\left|V_{n m}^{\mu}\right|^{2}}{E_{n}-E_{m}+K}=\frac{e^{2}}{4 n^{2}} \sum_{p_{2} P_{3}} \sum_{s=0}^{\infty} \sum_{l=1}^{2} \int \frac{d^{3} K}{K} \frac{\left|V_{s l, m}^{\mu}\right|^{2}}{E_{n}-m+K}=F(m, K), \\
& \frac{e^{2}}{4 \pi^{2}} \sum \int \frac{d^{3} K}{K} \frac{\left|V_{n m}^{\mu}\right|^{2}}{E_{n}-E_{m}+K}=\frac{e^{2}}{4 \pi^{2}} \sum_{P_{2,}, P_{3}} \sum_{s=0}^{\infty} \sum_{l=3}^{4} \int \frac{d^{3} K}{-K} \frac{\left|V_{s l, m}^{\mu}\right|^{2}}{\left|E_{n}\right|-m-K}=F(-m,-K),
\end{aligned}
$$

where

$$
F(m, k)=-\frac{e^{2}}{4 \pi^{2}} \int \frac{d^{3} K}{K} \sum_{s} \frac{\eta_{s}^{s} e^{-\eta}}{s !}\left\{-\frac{2 K_{3}^{2}+r^{2}}{2 E_{s+1}\left(E_{s+1}+m\right)\left(E_{s+1}-m+K\right)}+\frac{\left(E_{s}+m\right)^{2}-K_{3}^{2}-2 \frac{s^{2}}{\eta^{2}}}{2 E_{s}\left(E_{s}+m\right)\left(E_{s}-m+K\right)}\right\} .
$$

(Note that $E_{\mathrm{n}}$ is negative for the negative energy states but $E_{S}$ is always positive). Replacing $(s+1)$ by $s$ in the first term of $F(m, k)$ gives

$$
F(m, k)=\frac{e^{2}}{4 \pi^{2}} \int \frac{d^{3} K}{K} \sum_{s} \frac{\eta^{s} e^{-\eta}}{s !} g(s)
$$

where

$$
g(s)=\left\{\frac{m}{E_{s}}\left(1+\frac{s}{\eta}\right)-\frac{s}{\eta}\right\}\left(E_{s}-m+\kappa\right)^{-1} .
$$

Following Luttinger we can replace the sum over s to first order in e $\mathrm{H}_{0}$ by

$$
\begin{aligned}
& \sum_{s} \frac{n^{s} e^{-n}}{s !} g(s) \cong g(\eta)+\frac{\eta}{2} g^{\prime \prime}(\eta), \\
& g(\eta)=\left\{\frac{2 m}{\sqrt{m^{2}+k^{2}}}-1\right\}\left(\sqrt{m^{2}+k^{2}}-m+k\right)^{-1},
\end{aligned}
$$


which is independent of eH and gives the self energy of the free rest electron as listed in Table III. According to the method of mass renormalization this term is to be subtracted. It remains to calculate $g^{\prime \prime}(\eta) / 2$. If $s$ is written as $(\eta+\epsilon)$ theng" $(\eta) / 2$ is the coefficent of $\epsilon^{2}$ in the expansion of $g(s)$ in powers of $\epsilon$. When this has been found and $r^{2}$ replaced by its average value over the sphere, $\frac{2}{3} K^{2}$, the observable part of $\mathrm{F}(\mathrm{m}, \mathrm{K})$ is

$$
\begin{aligned}
F(m, k)=\frac{e^{2}}{\pi} \frac{e H_{0}}{m} \int_{0}^{\infty} m d k\left\{\frac{1}{2} \frac{m k^{2}-k^{3}}{\left(m^{2}+k^{2}\right)^{3 / 2}}+\frac{1}{6} \frac{5 k-m}{\left(m^{2}+k^{2}\right)^{3 / 2}}\right. \\
\\
+\left[\frac{k^{2}}{12 m\left(m^{2}+k^{2}\right)^{3 / 2}}+\frac{1}{6 m^{3}}\left(k-\sqrt{m^{2}+k^{2}}\right)\right]-\frac{1}{3 m^{2}}
\end{aligned}
$$

It should be noted that the last term alone in the integrand spoils the convergence of the integral. The convergence of the terms in square brackets together however is conditional on the signs of $\mathrm{K}$ and $\mathrm{m}$. 


\section{B. THE FHOTON NUMBER}

The matrix element $V_{n_{m}}$ is the emission element

$$
V_{n m}=-e\left(\frac{h c}{k L^{3}}\right)^{\frac{1}{2}}\left(u_{2}(-k) \alpha_{k, \gamma} u_{v_{0}}(0)\right),
$$

and

$$
W_{m}^{0}=E_{m}=m c^{2}, W_{n}^{0}=E_{n}+\hbar k c=I\left[\left(m c^{2}\right)^{2}+(\hbar k c)^{2}\right]^{\frac{1}{2}}+\hbar k c .
$$

In one-electron theory the sum extends over all spins in the intermediate state $n$. The expression for $\bar{N}$ is simplified by taking a factor thc out of the energies. This gives

$$
\begin{aligned}
\bar{N}=\frac{2 \pi}{L 3} \frac{e^{2}}{\hbar c} \sum_{k, \gamma} \frac{1}{k}\left\{\sum_{\nu=1}^{2} \frac{\left(u_{\nu_{0}}^{*}(0) \alpha_{k, \gamma} u_{\nu}(-k)\right)\left(u_{\nu}(-k) \alpha_{k, \gamma} u_{\nu_{\nu}}(0)\right)}{\left(\mu-\sqrt{\mu^{2}+k^{2}}-k\right)^{2}}\right. \\
+\sum_{\nu=3}^{4} \frac{\left(u_{\nu,}^{*}(0) \alpha_{k, \gamma} u_{\nu}(-k)\right)\left(u_{\nu}(-k) \alpha_{k, \gamma} u_{\nu_{0}}(0)\right.}{\left(\mu+\sqrt{\mu^{2}+k^{2}}-k\right)^{2}},
\end{aligned}
$$

where $\bar{\mu}=\frac{m c}{\hbar}$

Each sum over $\boldsymbol{v}$ can be extended over all four spin states after introducing the projection operators $C^{ \pm}(-k)$ defined by

$$
C^{ \pm}(k)=\frac{|E(k)| \pm H(k)}{2|E(k)|}=\frac{\sqrt{\mu^{2}+k^{2}} \pm(\alpha \cdot k+\beta \mu)}{2 \sqrt{\mu^{2}+k^{2}}}
$$

The value of $\bar{N}$ is averaged over both spins $\nu_{0}$ for the rest electron. Evaluation of $\bar{N}$ then requires the sum

$$
B(k)=\frac{1}{2} \sum_{\gamma} \frac{s_{p}\left(\alpha_{k, \gamma} C^{+}(-k) \alpha_{k, \gamma} C^{+}(0)\right)}{\left(\mu-\sqrt{\mu^{2}+k^{2}}-k\right)^{2}}+\frac{s_{p}\left(\alpha_{k, \gamma} C^{-}(-k) \alpha_{k, \gamma} C^{+}(0)\right)}{\left(\mu+\sqrt{\mu^{2}+k^{2}}-k\right)^{2}}
$$


This is easily evaluated using the fact that spurs containing an odd number of factors $\left(\beta, \alpha_{k}, \alpha_{\gamma}\right.$ vanish and using the commutation relations for $\alpha$ and $\beta$. The sum over polarizations gives simply a factor 2 .

$$
\begin{aligned}
2 B(k) & =\frac{\sqrt{\mu^{2}+k^{2}}-\mu}{\sqrt{\mu^{2}+k^{2}}\left(\mu-\sqrt{\mu^{2}+k^{2}}-k\right)^{2}}+\frac{\sqrt{\mu^{2}+k^{2}}+\mu}{\left.\sqrt{\mu^{2}+k^{2}}\left(\mu+\sqrt{\mu^{2}+k^{2}}-k\right)^{2}\right)} \\
& =\frac{1}{2 \mu^{2}} .
\end{aligned}
$$

With the sum over $k$ replaced by $\left(L^{3} /(2 \pi)^{3}\right) \int d^{3} k, \bar{N}$ reduces to

$$
\bar{N}=\frac{1}{2 \pi} \frac{e^{2}}{\hbar c}\left(\frac{\hbar}{m c}\right)^{2} \int_{0}^{\infty} k d k
$$

To make the transition to hole theory, the sum over intermediate states is first restricted by the exclusion principle to positive energy states. Contributions to $\overline{\mathrm{N}}$ from electrons in the Dirac sea would now have to be added. However since we are interested in the value of $\overline{\mathrm{N}}$ relative to the vacuum we need only to subtract the contributions from the sea which are disallowed by the presence of the one positive energy rest electron. These terms, which arise from a negative energy electron with initial momentum $\vec{k}$ which emits a photon of momentum $-\vec{k}$, give the sum

$$
\frac{2 \pi}{L^{3}} \cdot \frac{e^{2}}{\hbar c} \sum_{k, \gamma} \frac{1}{k} \sum_{\nu=3}^{4} \frac{\left(u_{\nu}^{x}(k) \alpha-k, \gamma u_{\nu,}(0)\right)\left(u_{\nu_{0}}^{x} \alpha-k, \gamma u_{\nu}(k)\right)}{\left(-\sqrt{\mu^{2}+k^{2}}-\mu-k\right)^{2}}
$$

Replacing $\vec{k}$ by $-\vec{k}$ and combining with the first term of $\overrightarrow{\mathbb{N}}$ in (3) gives 


$$
\begin{aligned}
\bar{N}_{\text {vact }}-\bar{N}_{\text {vac }}=\frac{2 \pi}{L^{3}} \frac{e^{2}}{\hbar C} \sum_{k, \gamma} \frac{1}{k}\left\{\frac{s p\left(\alpha_{k, \gamma} C^{+}(-k) \alpha_{k, \gamma} C^{+}(0)\right)}{\left(\mu-\sqrt{\mu^{2}+k^{2}}-k\right)^{2}}\right. \\
-\frac{s p\left(\alpha_{k, \gamma} C(-k) \alpha_{k, \gamma} C^{+}(0)\right)}{\left(\mu+\sqrt{\mu^{2}+k^{2}}+k\right)^{2}}
\end{aligned}
$$

It is worth noting that the transition to hole theory in effect simply changes the sign on the second term of $B(k)$ in (5) and changes the sign of the photon energy $k$ in the denominator of this term. This fact will be used again in Appendices $C$ and D. The new value of $B(k)$ is now obtained directly from Equation (6). It reduces to

$$
B(k)=\frac{\mu}{R\left(k+\sqrt{\mu^{2}+k^{2}}\right)}-\frac{\mu}{R\left(k+\sqrt{\mu^{2}+k^{2}}\right)}=0 \text {, }
$$

and $\overline{\mathbb{N}}$ vanishes in hole theory relative to the vacuum. 


\section{THE TRANSVERSE FIELDS}

The average values of the operators $\vec{E}_{\perp}(\vec{R})$ and $\vec{H}(\vec{R})$ from Equations (10), (11) and (3) are required up to second order in e. The only non-vanishing terms up to this order are the first order terms

$$
\begin{aligned}
& \vec{E}_{L}(\vec{R})=\sum_{\kappa}^{\prime} \frac{V_{K m}}{W_{m}^{0}-W_{n}^{0}} \int \psi_{m}^{o}(\vec{r}, q) \vec{E}_{1 o p}(\vec{r}+\vec{R}) \psi_{k}^{0}(\vec{r}, q) d \vec{r} d q+\text { conj. } \\
& \vec{H}(\vec{R})=\sum_{n}^{\prime} \frac{V_{K m}}{W_{m}^{0}-W_{n}^{0}} \int \psi_{m}^{0 *}(\vec{r}, q) \vec{H}_{o p}(\vec{r}+\vec{R}) \psi_{n}^{0}(\vec{r}, q) d \vec{r} d q+\text { conj. }
\end{aligned}
$$

Substituting for $V$ and $\vec{E}_{\text {lop }}$ from (4) and ( $I$ ) and carrying out the integration gives in one-electron theory

$$
\begin{aligned}
& \vec{E}_{1}(\vec{R})=-\frac{i e}{L^{3}} \sum_{\nu} \sum_{k, \gamma} \frac{\left(u_{0}^{*} u_{\nu}\right)\left(u_{\nu}^{*} \vec{e}_{\gamma} d \gamma u_{0}\right)}{E_{0}-E_{\nu}-\hbar k c} e^{i k \cdot R} h c+c o n j .
\end{aligned}
$$

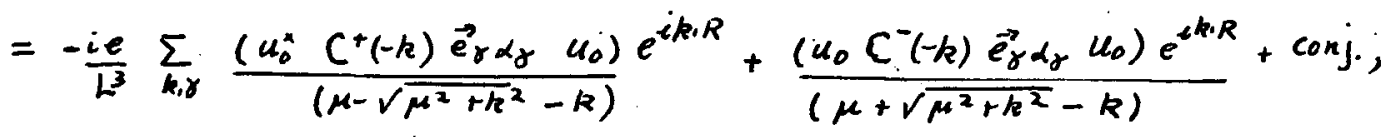

with $C^{5}(k)$ defined by Equation (4). A similar expression holds for $\vec{H}$ but $\vec{e}_{\gamma}$ is replaced by $\vec{k} \times \vec{e}_{\gamma} / k$. The sum over $\gamma$ gives

$$
\sum_{\gamma} \alpha_{\gamma} \overrightarrow{e_{\gamma}}=\vec{\alpha}-(\vec{\alpha} \cdot \vec{k}) \vec{k} / R^{2}, \quad \sum_{\gamma} \frac{\vec{k} \times \overrightarrow{e_{\gamma}} \alpha \gamma}{k}=\frac{\vec{k} \times \vec{\alpha}}{k}
$$


For a rest electron with spin up

$$
\begin{array}{r}
u_{0}=\left[\begin{array}{l}
1 \\
0 \\
0 \\
0
\end{array}\right], \quad\left(u_{0}^{x} \alpha u_{0}\right)=0, \quad\left(u_{0}^{x} \alpha \beta u_{0}\right)=0, \\
\left(u_{0}^{x} \alpha_{2} \alpha_{c} u_{0}\right)=1 ; i=1,2,3 . \\
\left(u_{0}^{x} \alpha_{1} \alpha_{2} u_{0}\right)=-\left(u_{0}^{x} \alpha_{2} \alpha_{1} u_{0}\right)=i\left(u_{0}^{x} \sigma_{3} u_{0}\right)=i, \\
\left(u_{0}^{x} \alpha_{2} \alpha_{3} u_{0}\right)=\left(u_{0}^{x} \alpha_{3} \alpha_{1} u_{0}\right)=0
\end{array}
$$

Using these we find

$$
\left(u_{0}^{*}(\alpha \cdot k) \vec{\alpha} u_{0}\right)=\vec{k}+i\left(k_{1} \vec{\epsilon}_{2}-k_{2} \vec{G}_{1}\right) \text { where } \overrightarrow{z_{1}} \vec{\epsilon}_{2} \vec{\epsilon}_{3} \text { are }
$$

the unit vectors of the $x, y, z$ coordinate system;

$$
\left(u_{0}^{*}(\alpha \cdot k)^{2} u_{0} \cdot\right) \vec{k}=k^{2} \vec{k} \text {. }
$$

$$
\begin{aligned}
\left.\left(u_{0}^{2}(\vec{\alpha} \cdot \vec{k}) \vec{k} \times \vec{\alpha}\right) \cdot u_{0}\right) & =i\left(\left(k_{1}^{2}+k_{2}^{2}\right) \vec{\epsilon}_{3}-k_{3} k_{2} \overrightarrow{\epsilon_{2}}-k_{3} k_{1} \vec{\sigma}_{1}\right), \\
& =i\left(k^{2} \vec{\epsilon}_{3}-k_{3} \vec{k}\right) .
\end{aligned}
$$

With these results the matrix elements for $\vec{E}$ and $\vec{H}$ are easily evaluated

$$
\begin{aligned}
& \vec{E}_{\perp}(\vec{R})=-\frac{2 \pi}{L^{3}} \text { e } \sum_{k}\left(k_{1} \vec{\epsilon}_{2}-k_{2} \vec{G}_{1}\right) \frac{\cos k \cdot R}{\sqrt{\mu^{2}+k^{2}}}\left\{\frac{1}{\mu-\sqrt{\mu^{2}+k^{2}}-k}-\frac{1}{\mu+\sqrt{\mu^{2}+k^{2}}-k}\right\} \\
& \vec{H}(\vec{R})=-\frac{2 \pi}{L^{3}}-e \sum_{k}\left(k \vec{\epsilon}_{3}-k_{3} \frac{\vec{k}}{k}\right) \frac{\cos k \cdot R}{\sqrt{\mu^{2}+k^{2}}}\left\{\frac{1}{\mu-\sqrt{\mu^{2}+k^{2}}-k}-\frac{1}{\mu+\sqrt{\mu^{2}+k^{2}}-k}\right\}
\end{aligned}
$$

Clearly $\vec{E}$ vanishes by symmetry, and $\vec{H}$ reduces to 


$$
\vec{A}(\vec{R})=\frac{e}{4 \pi^{2} \mu^{2}} \int d^{3} k\left(\vec{E}_{3}-k_{3} \frac{\vec{R}}{k}\right) \cos k \cdot R \text {. }
$$

To perform the integration over the angles take polar on $R$ and two auxiliary axes $S, T$ to complete the coordinate system, as shown in the diagram. For any vector $\vec{z}$, which can be chosen in the R,S plane without loss of generality, we have $\frac{1}{2 \pi} \int_{0}^{2 \pi}(k, \epsilon) \vec{k} d \varphi=\frac{1}{2 \pi} \int_{0}^{2 \pi}\left(k_{R} \epsilon_{R}+k_{s} \epsilon_{s}\right)\left(k_{R} \vec{R}_{1}+k_{s} \vec{s}_{1}+k_{r} \vec{t}_{1}\right) d \varphi$.

Expressing the components of $\vec{k}$ in terms of the

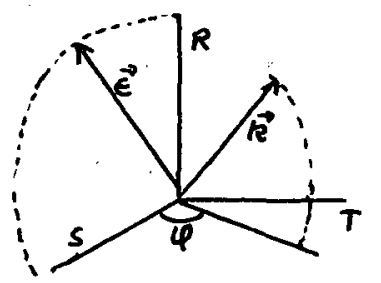
angles and carrying out the $\varphi$-integration then gives

$$
\frac{1}{2 \pi} \int_{0}^{2 \pi}(k \cdot \theta) \vec{k} d \varphi=k^{2} \cos ^{2} \theta\left(\vec{\epsilon} \cdot \vec{R}_{1}\right) \vec{R}_{1}+\frac{1}{2} k^{2} \sin ^{2} \theta\left[\vec{R}_{1} \times\left[\vec{\epsilon} \times \vec{R}_{1}\right]\right]
$$

This result is now used in Equation (20) but with $\left(k \cdot \epsilon_{3}\right)$ for $\mathrm{k}_{3}$

$$
\int d^{3} k=2 \pi \int_{0}^{\infty} k^{2} d k \int_{-1}^{r 1} d x \text { where } x=\cos \theta
$$

and

$$
\begin{aligned}
\vec{H}(\vec{R})= & \frac{e}{2 \pi \mu} \int_{0}^{\infty} d k k^{2} \int_{-1}^{\pi} \cos (k R x)\left\{\vec{\epsilon}_{3}^{\prime}-x^{2}\left(\vec{\epsilon}_{3} \cdot \vec{R}_{1}\right) \vec{R}_{1}-\frac{1}{2}\left(1-x^{2}\right)\left[\vec{R}_{1} \times\left[\vec{\epsilon}_{3}^{3} \times \vec{R}_{1}\right]\right]\right\} d x \\
= & \frac{e}{\pi \mu} \int_{0}^{\infty} d k k^{2}\left\{\frac{\sin k R}{k R} \vec{\epsilon}_{3}-\left[\frac{\sin k R}{k R}+\frac{2 \cos k R}{(k R)^{2}}-\frac{2 \sin k R}{(k R)^{3}}\right]\left(\vec{\epsilon}_{3} \cdot \vec{R}_{1}\right) \vec{R}_{1}\right. \\
& \left.+\left[\frac{\cos k R}{(k R)^{2}}-\frac{\sin k R}{(k R)^{3}}\right]\left[\vec{R}_{1} \times\left[\vec{\epsilon}_{3} \times \vec{R}_{1}\right]\right]\right\} .
\end{aligned}
$$




$$
\begin{gathered}
\vec{H}(\vec{R})=\frac{e}{\pi \mu R^{3}} \int_{0}^{\infty} y d y\left\{\sin y \vec{\epsilon}_{3}-\left[\sin y+\frac{2 \cos y}{y}-\frac{2 \sin y}{y^{2}}\right]\left(\vec{G}_{3} \cdot \vec{R}_{1}\right) \vec{R}_{1}\right. \\
+\left[\frac{\cos y}{y}-\frac{\sin y}{y}\right]\left[\vec{R}_{1} \times\left[\vec{\epsilon}_{3} \times \vec{R}_{1}\right]\right] .
\end{gathered}
$$

The integrals appearing in (25) have the values

$$
\int_{0}^{\infty} y d y \sin y=\int_{0}^{\infty} d y \cos y=0 ; \int_{0}^{\infty} d y \frac{\sin y}{y}=\frac{\pi}{2},
$$

and $\vec{H}(\vec{R})$ becomes

$$
\vec{H}(\vec{R})=\frac{1}{R^{3}}\left\{2\left(\vec{M} \cdot \vec{R}_{1}\right) \vec{R}_{1}+\left[\vec{R}, \times\left[\overrightarrow{R_{1}} \times \vec{M}\right]\right] ; \vec{M}=\frac{e \hbar}{2 m c} \vec{\epsilon}_{3} .\right.
$$

The transition to hole theory is made as in Appendix B starting with Equation (19). The electric field vanishes as in one-electron theory while

$$
\begin{aligned}
\vec{H}(\vec{R}) & =-\frac{2 \pi}{L^{3}} \text { e } \sum_{k}\left(k \vec{\epsilon}_{3}-k_{3} \frac{\vec{r}}{k}\right) \frac{\cos k \cdot R}{\sqrt{\mu^{2}+h^{2}}}\left\{\frac{1}{\mu-\sqrt{\mu^{2}+k^{2}-k}}+\frac{1}{\mu+\sqrt{\mu^{2}+k^{2}+k}}\right\} \\
& =-\frac{2 \pi}{L^{3}} \text { e } \sum_{k}\left(k \overrightarrow{\epsilon_{3}}-k_{3} \frac{\vec{C}}{k}\right) \frac{\cos k \cdot R}{\sqrt{\mu^{2} r k^{2}}} \mu
\end{aligned}
$$

This result differs from the corresponding 'Equation (20) by a factor $\mu\left(\mu^{2}+k^{2}\right)^{-\frac{1}{2}}$, so that Equation (25) can be used with the integrand multiplied by this factor.

$$
\begin{aligned}
\vec{H}(\vec{R})=\frac{e}{\pi} \frac{1}{R^{2}} \int_{0}^{\infty} \frac{y d y}{\sqrt{\mu^{2} R^{2}+y^{2}}}\{ & \sin y \vec{\epsilon}_{3}-\left[\sin y+2 \frac{\cos y}{y}-\frac{2 \sin y}{y z}\right]\left(\epsilon_{3} \cdot R_{1}\right) \vec{R}_{1} \\
& +\left[\frac{\cos y}{y} y-\frac{\sin y}{y^{2}}\right]\left[\vec{R}_{1} \times\left[\vec{\epsilon}_{3} \times \vec{R}_{1}\right]\right] .
\end{aligned}
$$


The following integrals are required

$$
\begin{aligned}
& \int_{0}^{\infty} \frac{d y \sin y}{\sqrt{a^{2}+y^{2}}}=a K_{0}(a), \quad \int_{0}^{\infty} \frac{\cos y}{\sqrt{a^{2}+y^{2}}} d y=K_{0}(a) \\
& \int_{0}^{\infty} \frac{\sin y d y}{y \sqrt{a^{2}+y^{2}}}=\int_{0}^{1} d x \int_{0}^{\infty} \frac{\cos x y}{\sqrt{a^{2}+y^{2}}} d y=\int_{0}^{1} d x K_{0}(a x),
\end{aligned}
$$

where $K_{\nu}(a)$ is the Hankel function of the third kind. In the last integral parametric integration over $x$ was used to removed. the factor $\mathrm{y}^{-1}$ and the order of integration was then reversed. With these integrals substituted into (29) Equation (17) of Section VI is obtained.

\section{THE COULOMB FIELD}

The expectation value of $\vec{E}_{\|}(R)$ is

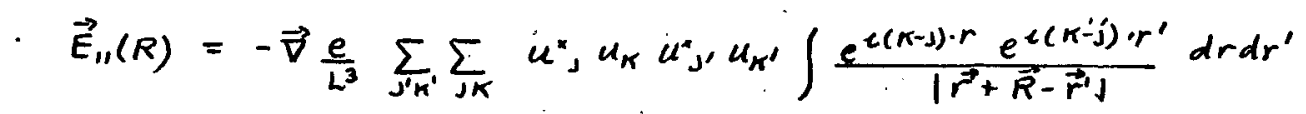

$$
\times\left(\psi^{\dot{0}}, a_{j}^{x} a_{k} a_{j}^{x}, a_{\kappa^{\prime}} \psi^{0}\right)
$$

In one-electron theory

$$
\left(\Psi^{0}, a_{j}^{k} a_{n} a_{j}^{k} a_{k^{\prime}} \psi^{0}\right)=\left(\Psi^{0}, a_{j}^{x}\left(\delta_{k j}-a_{j,}^{k} a_{k}\right) a_{k^{\prime}} \psi^{0}\right),
$$

by using the anticommutation relations (22) of section VI. Since there is only one electron to be annihilated we have 
$-55-$

$$
\left(\Psi^{\circ}, a_{\jmath}^{*} a_{k^{\prime}} \Psi^{\circ}\right) \delta_{k J^{\prime}}=\delta_{j k^{\prime}} \delta_{k J^{\prime}} \delta_{k^{\prime} 0},
$$

and

$$
\begin{aligned}
\vec{E}_{11}(R) & =-\vec{\nabla} \frac{e}{L^{3}} \sum_{k}\left(u_{0}^{*} u_{k} u_{k}^{*} u_{0}\right) \int \frac{e^{<k \cdot\left(r-r^{\prime}\right)}}{\left|\vec{r}+\vec{R}^{\prime}-\vec{r}^{\prime \prime}\right|} d \vec{r} d \vec{r}^{\prime}, \\
& =-\vec{\nabla} \frac{e}{L^{3}} \int \frac{d \vec{r} d \vec{r}^{\prime}}{\left|\vec{r}+\vec{R}-\vec{r}^{\prime \prime}\right|} \delta\left(\vec{r}-\vec{r}^{\prime}\right) . \\
\vec{E}_{1 \prime}(R) & =-\vec{\nabla} \frac{e}{R} .
\end{aligned}
$$

The transition to hole theory is made as in Appendix B:

$$
\begin{aligned}
& \vec{E}_{1}(R)=-\vec{D} \frac{e}{L^{*}} \int \frac{\left.d \vec{r}^{2} d \vec{r}^{*}\right)}{\left.\vec{r}+\vec{R}-\vec{r}^{\prime}\right)}\left\{\sum_{\kappa} \sum_{\nu=1}^{2}\left(u_{0}^{*} u_{\nu} u_{\nu}^{*} u_{0}\right) e^{i k \cdot\left(r r^{\prime}\right)}-\sum_{\kappa^{\prime}} \sum_{\nu^{\prime}=3}^{4}\left(u_{\nu}^{*} u_{0}\right)\left(u_{0}^{*} u_{\nu^{\prime}}\right) e^{-2 k\left(r, r^{\prime}\right)}\right\}, \\
& =-\frac{\vec{\nabla} e}{(2 \pi)^{3} L^{2}} \int \frac{d r d r^{\prime}}{\left|\vec{r}+\vec{R}^{\prime}-\vec{r}^{\prime}\right|} d^{k}\left\{u_{0}^{*} C^{*}(k) u_{0} e^{i k \cdot\left(r r^{\prime}\right)}-u_{0}^{x} C^{-}(k) u_{0} e^{-i k \cdot(r-r)}\right\} \text {, }
\end{aligned}
$$

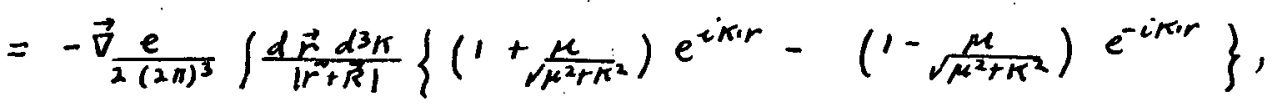

$$
\begin{aligned}
& =-\vec{\nabla} \frac{e}{2 \pi^{2}} \int \frac{d^{3} k}{k^{2}} \frac{\mu}{\sqrt{\mu^{2}+k^{2}}} \cos k \cdot R, \\
& =-\vec{\nabla} \frac{e \mu}{R} \frac{2}{\pi} \int_{0}^{\infty} \frac{d K}{\pi} \underset{\sin K R}{\sqrt{\mu^{2}+K^{2}}} .
\end{aligned}
$$

Formula (31) can now be used:

$$
\vec{E}_{11}(R)=-\vec{\nabla} \frac{2}{\pi} \frac{e}{\lambda_{0}} \int_{0}^{1} d x K_{0}\left(\frac{R}{\lambda_{0}} x\right) .
$$




\section{REFERENCES}

1. I. Waller, Zeits.f.Phys. 63, 673,(1930)

2. P.A.M. Dirac, Proc.Roy.Soc.Lon. Al17, 610, (1928)

3. P.A.M. Dirac, Proc.Roy.Soc.Lon. Al14, 243,(1927)

4. W. Heisenberg and W.Pauli, Zeits.f.Phys.56, 1, (1929) $59,168,(1930)$

5. R. Oppenheimer, Phys.Rev. 35, 461, (1930)

6. P.A.M. Dirac, Proc.Roy.Soc.Lon., Al26, 360, (1929)

7. V. Weisskopf, Zeits.f.Physik, 89, 27,(1934)

8. V. Weisskopf, Phys.Rev., 56, 72, (1939)

9. W.E. Lamb Jr., Reports of Progress in Physics, 14,20,(1951) (Review article and bibliography)

10. S. Pasternack, Phys. Rev. 54, 113,(1938)

11. W.E. Lamb Jr. and R.C. Retherford, Phys.Rev. 22, 241, $(1947) ; 25,1325 \mathrm{~A},(1949)$

12. H.A. Bethe, Phys.Rev. 72, 339, (1947)

13. H.A. Bethe et al., Phys.Rev. 27, 370, (1950)

N. M. Kroll and W.E. Lamb Jr. Phys.Rev. 75, 388, (1949)

J.B. French and V. Weisskopf,Phys.Rev. 25, 1240, (1949)

14. J. Schwinger, Phys.Rev. 23, 416, (1948)

15. G. Breit, Phys.Rev. 22, 984,(1947)

16. S. Tomonaga, Prog.Theor.Phys. 1,27 (1946); 2,101,198, $(1.947) ; 3,1,101,(1948)$

17. J. Schwinger, Phys. Rev. 24,1439 (1948); 25, 651,(1949)

18. F.J. Dyson, Phys.Rev. 25, 486(1949) and 25,1736, (1949)

19. R.P.Feynman, Phys.Rev. 26, 749, (1949)

20. R. P.Feynman, Phys.Rev. 26, 769, (1949)

21. R.P. Feynman, Phys.Rev. 80, 440, (1950) 
22. P.N. Daykin, Can.Jour.of Phys. 22, 459, (1951)

23. S. Koenig, A.G. Prodell and P.Kusch, Phys.Rev. 83 $687,(1951)$

24. Karplus and Kroll, Phys.Rév. 27, 536,(1950)

25. J.A."Wheeler and R.P. Feynman; Rev.Mod.Phys. 17, i57, (1945)

26. P.A.M. Dirac, Proc.Roy.Soc.Lon. Al67, 1, (1942)

27. W.Pauli, Rev.Mod.Phys. 15, 175, (1943)

28. T. Muto and K. Inone, Prog.Theor.Phys. 5, 1033,(1949)

29. G. Breit, Phys.Rev. 34, 553, (1929)

30. C. Stueckelberg, Helv.Phys.Acta.11, 225,(1938)

31. J. Schwinger, Phys.Rev. 82, 664,(1951) Appendix

32. J.M. Luttinger, Phys.Rev: 24, 893,(1948)

33. C.G. Darwin, PhilMag. 39, 537,(1920)

34. H.A. Bethe and E. Fermi, Zeits.F.Phys.27, 296,(1933)

35. W. Heitler, Quantum Theory of Radiation, Oxford Univ. Press, p. 106. 